\title{
Addressing challenges of heterogeneous tumor treatment through bispecific protein-mediated pretargeted drug delivery
}

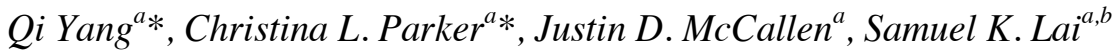

${ }^{a}$ Division of Molecular Pharmaceutics, Eshelman School of Pharmacy; ${ }^{b}$ UNC/NCSU Joint Department of Biomedical Engineering; University of North Carolina at Chapel Hill

[*co-first authors]

Corresponding Author:

Samuel K. Lai

Division of Molecular Pharmaceutics

University of North Carolina at Chapel Hill

Marsico 4213, 125 Mason Farm Road

Fax: (+001)-919-843-1232

E-mail: lai@unc.edu

\begin{abstract}
Tumors are frequently characterized by genomically and phenotypically distinct cancer cell subpopulations within the same tumor or between tumor lesions, a phenomenon termed tumor heterogeneity. These diverse cancer cell populations pose a major challenge to targeted delivery of diagnostic and/or therapeutic agents, since the conventional approach of conjugating individual ligands to nanoparticles is often unable to facilitate intracellular delivery to the full spectrum of cancer cells present in a given tumor lesion or patient. As a result, many cancers are only partial suppressed, leading to eventual tumor regrowth and/or the development of drug-resistant tumors. Pretargeting (multistep targeting) approaches, which involves administering 1) a cocktail of bispecific proteins that
\end{abstract}


can collectively bind to the entirety of a mixed tumor population followed by 2) nanoparticles containing therapeutic and/or diagnostic agents that can bind to the bispecific proteins accumulated on the surface of target cells offers the potential to overcome many of the challenges associated with drug delivery to heterogeneous tumors. Despite its considerable success in improving the efficacy of radioimmunotherapy, the pretargeting strategy remains underexplored for a majority of nanoparticle therapeutics applications, especially for targeted delivery to heterogeneous tumors. In this review, we will present concepts in tumor heterogeneity, the shortcomings of conventional targeted systems, lessons learned from pretargeted radioimmunotherapy, and important considerations for harnessing the pretargeting strategy to improve nanoparticle delivery to heterogeneous tumors.

Keywords: pretargeting, tumor heterogeneity, nanoparticles, cancer targeting, bispecific proteins, drug delivery

\section{Introduction}

Targeted drug delivery for cancer offers the potential to significantly improve the therapeutic index of anticancer agents by increasing drug concentration at tumor sites while reducing side effects and toxicity in nontargeted tissues. A long-standing approach in the field has been to exploit the leaky tumor vasculature in tumor tissues by encapsulating therapeutic cargo into nanoparticles that remain sufficiently stable when introduced to the systemic circulation in order to reach and extravasate into cancer tissues. To further facilitate selective delivery into cancer cells, many researchers have functionalized nanoparticles with ligands that bind specific receptors on cancer cells, a strategy commonly referred to as "active" targeting [1]. Unfortunately, the accumulation of both ligand-free and ligand-conjugated systems in tumors are generally modest at best, limiting the efficacy of various therapies against cancer $[2,3]$.

Due to advances in the genetic and phenotypic analysis of tumors, tumor heterogeneity has recently emerged as yet another biological barrier that could limit efficient distribution of ligand-conjugated nanoparticles to tumor sites [3]. Tumor heterogeneity refers to the highly variable expression of target receptors, both intertumorally between patients or different tumors and intratumorally within a given tumor, and has been reported for a wide range of human tumors $[4,5]$. Actively targeted drug carriers, which typically consist of single-ligand nanoparticles, are unable to effectively bind and internalize into the full spectrum of tumor cells present in any particular tumor. 
Incomplete drug delivery to all cancer cell subpopulations typically results in only partial suppression of the cancer and eventually leads to tumor regrowth and/or the development of drug-resistant tumors [6]. Thus, targeting strategies that can directly address the challenges associated with tumor heterogeneity and enable effective delivery of nanoparticles are sorely needed.

One promising targeting strategy is to decouple molecular homing and delivery of therapeutics into two separate steps. This approach involves first introducing bispecific proteins (BsPs) that can specificially bind (i.e., "pretarget") cancer cells, followed by the administration of a drug-carrying effector such as a nanoparticle that can be captured by the BsPs accumulated on the surface of tumor cells (Figure 1). By introducing multiple distinct BsPs, a single effector nanoparticle could in theory bind with molecular specificity to the full diversity of cancer cells present in any particular tumor. In this review, we will discuss the concept of, important considerations for, and key challenges associated with exploiting the pretargeted strategy to enhance the delivery of therapeutics to heterogeneous tumors. 
a

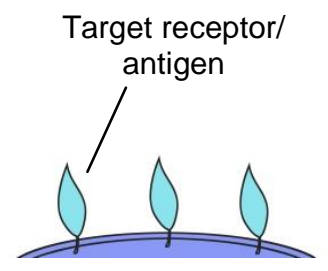

Tumor cell
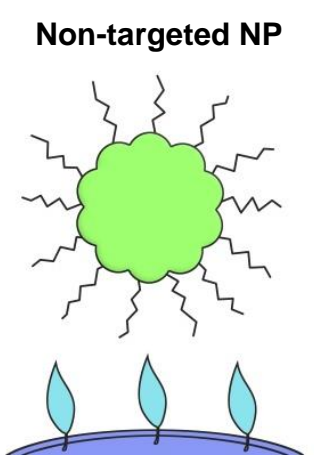

RIT

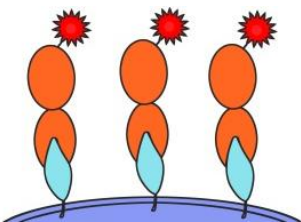

C Active targeted NP

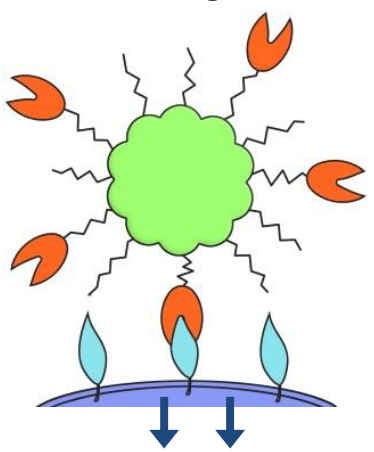

d

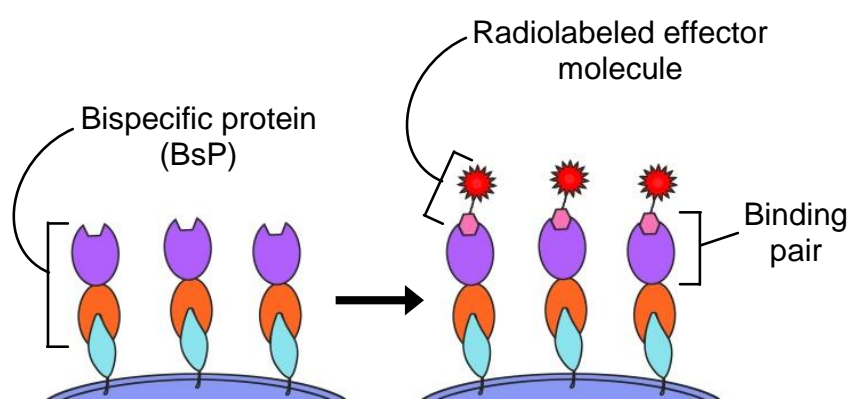

e

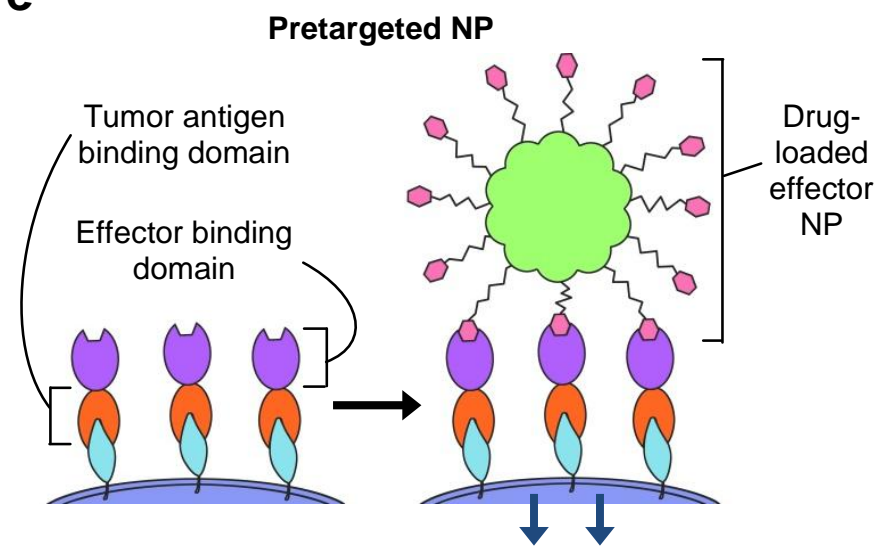

Figure 1. Strategies for the delivery of nanoparticle drug carriers and/or radioisotopes to tumor cells include a) non-targeted, b \& c) directly targeted (1-step), and $\mathbf{d} \& \mathbf{e}$ ) pretargeted (multistep) approaches. a) Passively targeted nanoparticles coated solely with stealth polymers typically do not exhibit specific interactions with tumor cells. b) Radioimmunotherapy (RIT) uses radiolabeled tumor receptor-specific antibodies to deliver therapeutic doses of radiation to target cells. c) Modification with receptor-specific ligands allows the active targeting of nanoparticles (NPs) to tumor cells, which commonly induces receptor-dependent internalization. d) Pretargeted radioimmunotherapy (PRIT) splits tumor targeting and radioisotope delivery into sequential steps: 1) binding of bispecific proteins (BsPs) to target receptors and 2) binding of radiolabeled effector molecules to the BsPs. e) For pretargeted drug delivery systems, 1) bispecific proteins (BsPs) bind target receptors, and 2) a drug-loaded effector nanoparticle binds to the BsPs, which should ideally result in internalization.

\section{Conventional cancer targeting strategies: passive targeting}

In 1986, Matsumura and Maeda discovered that macromolecules can preferentially accumulate in tumors due to anatomical and pathophysiological differences between solid tumors and healthy tissue [7-9]. Specifically, tumors initiate extensive angiogenesis to maintain their rapid growth, but the newly formed blood vessels display 
abnormal architecture including fenestrated endothelial lining of vessel walls [8-10]. The more permeable tumor vasculature then allows macromolecules and nanoparticles to extravasate from the bloodstream and accumulate in the tumor $[9,10]$. Presumably poor lymphatic drainage further permits enhanced retention of drug delivery systems within tumors $[8,10]$. The combination of leaky tumor vasculature and impaired lymphatic drainage constitute the phenomenon termed the enhanced permeability and retention (EPR) effect.

Harnessing the EPR phenomenon simply requires nanoparticles to (i) fall within an appropriate size range and (ii) evade rapid elimination by the mononuclear phagocytic system (MPS). While smaller nanoparticles can naturally extravasate more efficiently than larger nanoparticles, most studies suggest the tumor vasculature in mouse xenografts can permit extravasation of nanoparticles ranging from 10 to $200 \mathrm{~nm}$ in diameter [9, 11-13] with some studies reporting EPR of particles up to $500 \mathrm{~nm}$ in diameter [10,14]. In addition to size, prolonged circulation kinetics also directly improve the extent of nanoparticle extravasation through leaky tumor blood vessels by maximizing the number of times a nanoparticle will pass through the tumor vasculature $[8,14]$. Polyethylene glycol (PEG) was among the first "stealth" polymers used to extend liposome and other nanoparticle circulation times by minimizing opsonin adsorption and nanoparticle elimination by MPS cells, and PEGylation is the most widely adopted strategy to enhance nanoparticle tumor uptake via EPR [8-10, 14]. Other coating polymers used to improve particle circulation profiles, and thereby exploit the EPR effect, include flexible, hydrophilic polysaccharides such as dextran, hyaluronic acid, and chitosan [15, 16]; synthetic polymers such as polyvinyl alcohol $[14,15]$ and polyvinylpyrrolidone [14, 15]; zwitterionic polymers [15, 16]; and polyoxazolines [16]. Indeed, dextran-, hyaluronic acid-, chitosan-, and N-(2-hydroxylpropyl) methylacrylamide (HPMA)-coated particles all exhibited improved EPR-mediated tumor accumulation due to prolonged circulation $[16,17]$. Since nanoparticles of the appropriate size and with MPS-resistant surface chemistry can naturally achieve a low to moderate level of tumor targeting without using specific ligands, these non-molecularly targeted systems are frequently classified as passively targeted.

It is important to note that the EPR effect is highly variable and may not be readily exploitable for all tumors [18]. For example, hepatocellular and renal cell carcinomas are characterized by high vascular density and exhibit increased EPR effects compared to low vascular density pancreatic and prostate cancers that demonstrate diminished EPR effects [18]. Additionally, EPR of drug carriers is not observed homogenously throughout 
individual tumors, as the central foci of tumors tend to be characterized by necrotic [18], hypoxic [18], and hypovascular areas [8] that do not display the EPR effect $[1,7]$. EPR heterogeneity has also been reported between primary tumor and metastases [1]. Therefore, harnessing EPR to enhance therapeutic responses in the clinic requires an improved understanding of how tumor heterogeneity impacts the EPR effect both within and between tumors $[1,9,18-20]$.

\section{Conventional cancer targeting strategies: active targeting}

To further improve nanoparticle-based delivery to cancer cells, numerous investigators have developed nanoparticles decorated with ligands specific to receptors overexpressed on cancer cells, an approach generally termed active targeting [1]. Ligands on actively targeted systems are typically grafted to the distal end of polymer chains that are used to coat the particles and provide prolong circulation kinetics [14]. These systems are presumed to effectively extravasate from the tumor vasculature based on the underlying stealth polymer coating, while the presence of ligands can facilitate nanoparticle binding to and subsequent internalization into specific tumor cells expressing the corresponding receptor $[14,21]$. Actively targeted systems were thought to directly address the shortcoming of inefficient cellular uptake of passively targeted systems [10, 14]. Numerous targeting ligands have been utilized to actively target nanoparticles to cancer cells, including antibodies and antibody fragments, aptamers, peptides, proteins, sugars, and low molecular weight ligands such as folate [1, 14, 21, 22].

Unfortunately, active targeting systems face several challenges that may limit their efficacy in practice. The target cell surface receptors must be highly overexpressed or selectively expressed solely on malignant cells, as opposed to healthy cells, to maximize tumor-specific delivery [22-24]. Additionally, the choice and density of ligand are critical to optimizing the effect of the targeting moiety [23]. Greater ligand density was previously assumed to enhance nanoparticle targeting to tumors in vivo due to generally observed improvements in cancer cell uptake in vitro [23]. Nevertheless, an increasingly number of studies have shown that maximal accumulation of nanoparticles in tumors in vivo is typically achieved with an intermediate ligand density [23, 25-28]. For example, increasing the surface aptamer density on polymeric nanoparticles actually resulted in reduced tumor accumulation and increased particle distribution in the liver [25]. The poor in vivo performance of particles with high ligand densities was attributed to ligand shielding or adulteration of the underlying stealth polymer coat, leading to rapid MPS clearance and a reduction in the fraction of particles that can reach and extravasate into tumors [23, 24]. 


\section{Tumor heterogeneity and implications for targeted drug delivery systems}

Variations in accumulated genetic mutations, which can be further exacerbated by alterations in the local tumor microenvironment, frequently lead to genomically distinct subclonal populations within the same tumor or between tumor lesions. This in turn creates a phenomenon termed tumor heterogeneity, which describes the functional and phenotypic profile differences between cancer cells such as cellular morphology, gene expression, metabolism, motility, proliferation, level of drug resistance, and metastatic potential. Tumor heterogeneity (Figure 1) encompasses both (i) intertumoral heterogeneity, which describes differences between tumors in an individual patient as well as clinical response differences between patients with the same tumor subtype, and (ii) intratumoral heterogeneity, which refers to the genetic, epigenetic, and phenotypic features that vary within malignant cell populations of the same tumor mass [29]. Intratumoral heterogeneity is further classified into spatial heterogeneity, which refers to differences between distinct anatomical regions or individual cells within a tumor, and temporal heterogeneity, which refers to changes in a tumor's molecular profile and receptor expression over time. An example of intratumoral spatial heterogeneity is the highly discordant HER2 expression observed in different areas within a single biopsy from HER2-positive metastatic breast cancer patients (Figure 3a) [30]. Temporal heterogeneity can be observed for relapsed lesions that exhibit a disparate molecular profile, compared to their original tumor.

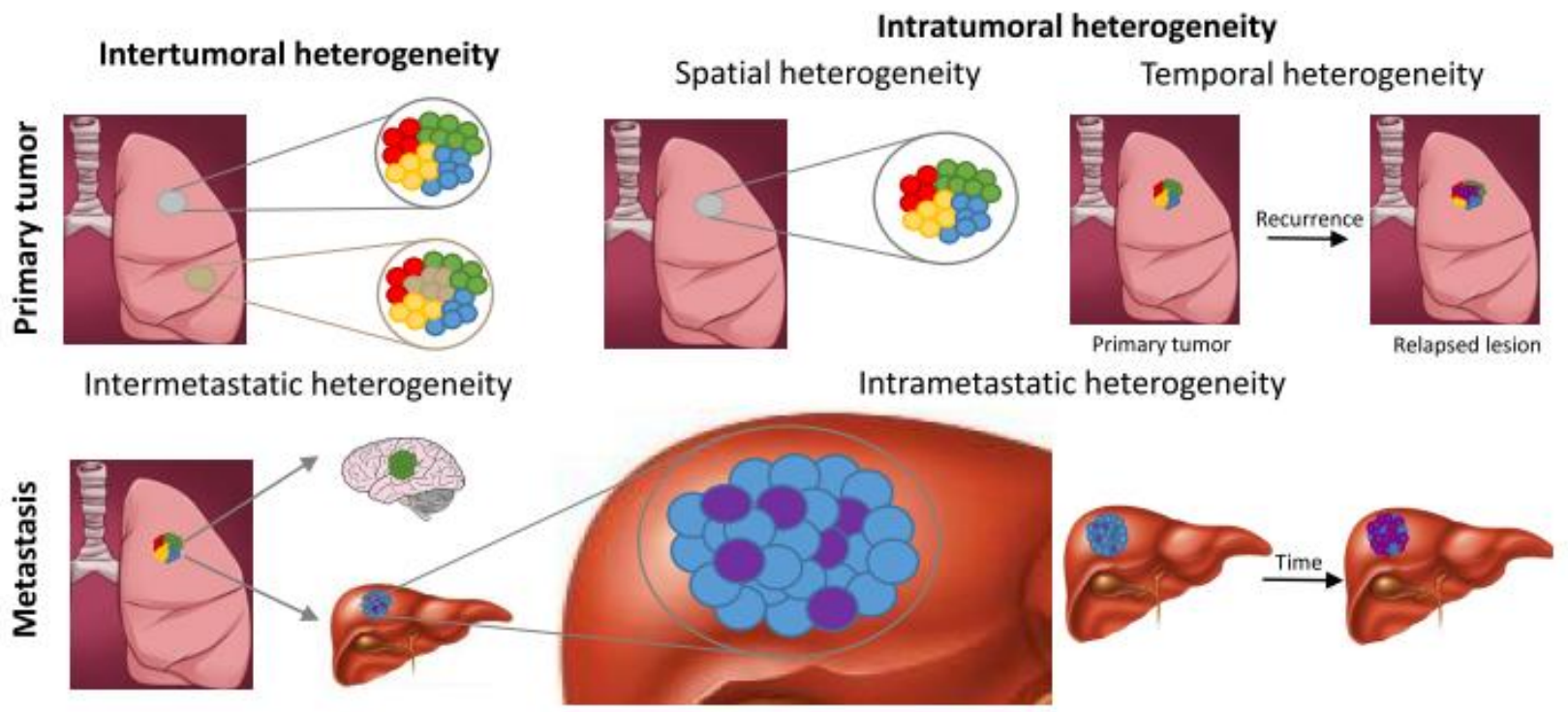

Figure 2. Different types of tumor heterogeneity. Spatial heterogeneity refers to differences between distinct anatomical regions or individual cells within a tumor, while temporal heterogeneity illustrates changes in a tumor's 
molecular profile over time. Intermetastatic heterogeneity arises from distinct subclonal populations in the primary tumor, and intrametastatic heterogeneity reflects the discordant molecular profiles of cells within individual metastases.
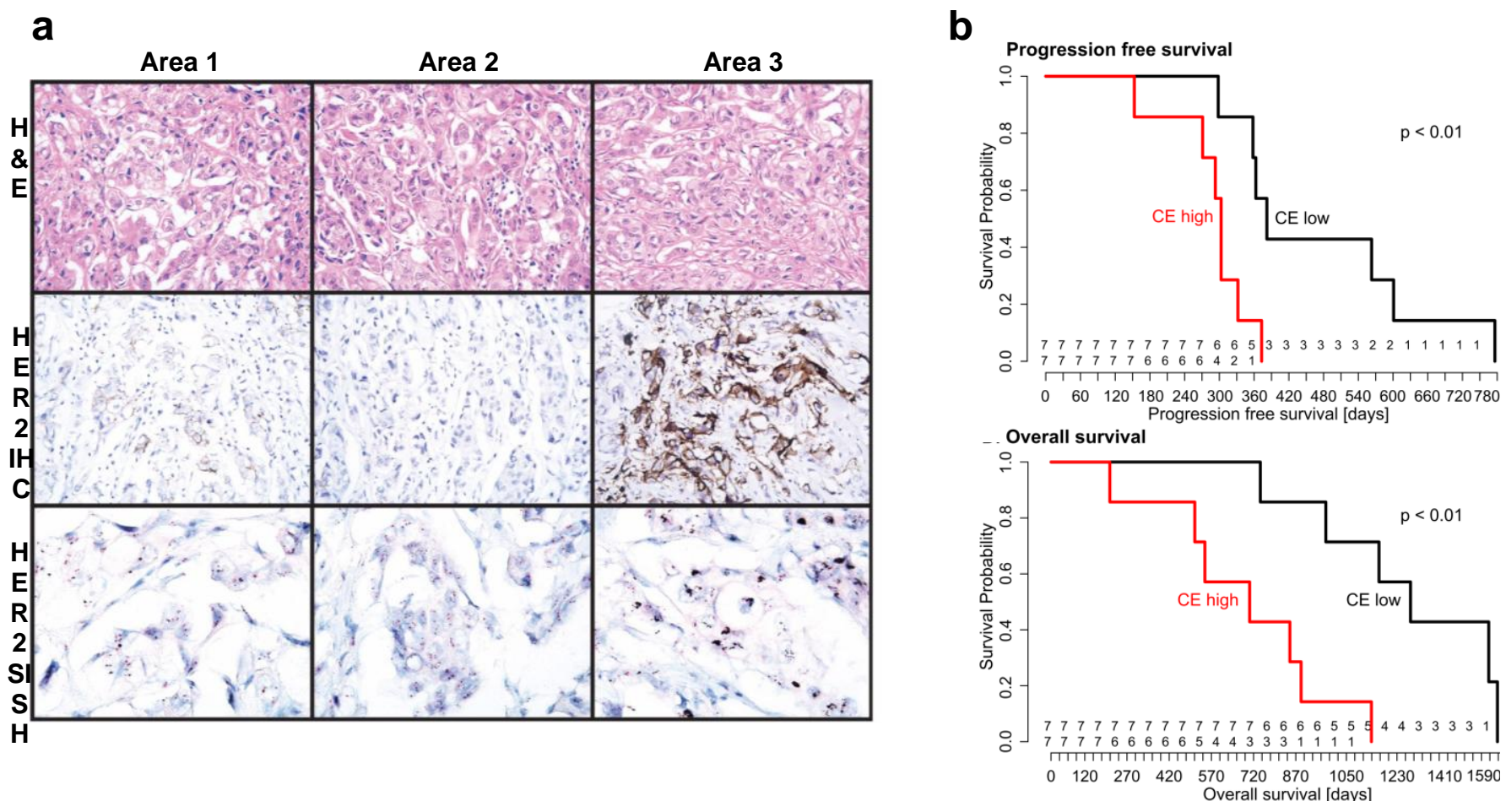

Figure 3. a) Spatial heterogeneity in HER2 expression between three different areas of an invasive ductal carcinoma biopsy sample. HER2 amplification was confirmed using immunohistochemistry (IHC) and silver in situ hybridization (SISH). H\&E, ×200; IHC, ×200; SISH, ×400[30]. b) Progression free survival (top) and overall survival (bottom) of high-grade serous ovarian cancer patients treated with platinum-based chemotherapy and surgery, stratified by degree of clonal expansion (CE). CE reflects the accumulation of mutations that promote cell expansion into varying subclonal populations from the original cell. Higher CE is correlated with divergent subclonal populations and thus greater tumor heterogeneity [31].

In addition to morphological and spatiotemporal variations within the same tumor or between primary tumors, tumor heterogeneity can also directly result from metastasis. Metastatic heterogeneity (Figure 2) has been observed to include (i) discordant biomarker or receptor expression between metastases arising from distinct subclonal populations in the primary tumor ("intermetastatic" heterogeneity) and (ii) heterogeneity within 
individual metastases ("intrametastatic" heterogeneity), which may have a substantial impact on therapeutic outcome [5, 29, 32-34]. For example, Gerlinger et al. reported a case of intrametastatic heterogeneity in which significant changes in the mutational profiles of spatially separated biopsy samples from primary renal-cell carcinomas and metastases were identified using next-generation sequencing [35]. Additionally, Albino et al. observed intermetastatic heterogeneity in a melanoma patient whose multiple metastases displayed contrasting morphologies and surface antigen expression [34]. Other studies have also investigated variable estrogen, progesterone, and HER2 receptor expression between primary breast tumors and metastases, with discordance rates that varied greatly from $18 \%$ to $54 \%[32,36,37]$. Additional types of heterogeneity include non-genetic phenotypic and functional heterogeneity [37] and tumor microenvironment heterogeneity [32, 38]. Because tumor cells interact with their environment, tumor microenvironment heterogeneity exerts a crucial influence on disease progression. For example, the heterogeneous distribution of stromal cells, extracellular matrix organization, and especially hypoxic regions within the tumor microenvironment may promote metastasis and development of drug resistance [32].

Tumor heterogeneity has been reported in a wide range of human tumors such as breast [30, 32], non-small cell lung [39], ovarian [31, 32, 40, 41], prostate [32, 42], and lymphoma [43] and poses a significant challenge for diagnosis, prognosis, and efficacy of molecularly-targeted therapies (Figure 3b) [31,44] . The presence of heterogeneous cancer cell populations within tumors will likely limit the efficacy of any therapeutics targeted against any single tumor-associated receptor, leading to poor/varied outcomes, including cancer recurrence and therapeutic resistance [4, 29]. For example, the heterogeneous expression of programmed death 1 (PD-1) was reported in two distinct T-cell subpopulations and differentially impacted survival in patients with follicular lymphoma [43]. Similarly, heterogeneous HER2 expression in breast cancer has prompted treatment stratification in the clinic based on receptor expression [32]. Indeed, intratumoral HER2 heterogeneity, both genetic and spatial, affected the trastuzumab treatment responses and survival of patients with HER2-positive metastatic breast cancer [30]. Only a small fraction of trastuzumab-treated patients achieved complete disease eradication, and the majority of patients developed relapsed tumors that were resistant to trastuzumab therapy due to the proliferation of HER2negative breast cancer cells. 
In addition to therapy with monoclonal antibodies such as trastuzumab, variable target receptor expression in heterogeneous tumors also presents a critical bottleneck for actively targeted drug delivery systems. The common active targeting approach, in which drug-loaded particles are surface modified with a single ligand group, cannot target and facilitate intracellular delivery to the full diversity of malignant cells. One potential strategy is the administration of a cocktail of single-ligand particles. Unfortunately, this would pose considerable challenges and substantial cost burden in the context of particle formulation and complexity in clinical evaluation [24], which has generally limited particles to one or two distinct targeting ligand groups. More importantly, a single universal targeted nanoparticle cocktail for all patients is unlikely to succeed due to interpatient heterogeneity; inadequate levels or the complete lack of corresponding target cells for a significant fraction of the ligand-modified particles could lead to increased hepatic and splenic biodistribution and, correspondingly, reduced tumor accumulation. Alternatively, multiple different targeting ligands could be theoretically conjugated onto the surface of a single nanoparticle. However, as discussed above, increased density of ligands beyond a particular threshold will likely trigger rapid MPS clearance of the particles.

\section{Pretargeted radioimmunotherapy (PRIT)}

The discovery that human tumor-associated antigens could be used as targets for antibodies to differentiate tumors from normal tissue helped spawn the field of monoclonal antibody (MAb)-based immunotherapy of cancer. The multiple applications of cancer immunotherapy include radioimmunotherapy (RIT) (Figure 1b), which uses radioisotope-conjugated Mabs to treat radiosensitive tumors such as non-Hodgkin's lymphoma (NHL) [45]. Unfortunately, the therapeutic efficacy of RIT is limited by the long circulatory half-life of many MAbs, as well as high non-specific deposition of the MAbs in normal organs, resulting in low tumor-specific delivery of radiation and significant toxicity [46].

To overcome the shortcomings of radioimmunotherapy (RIT), many researchers have adopted a multistep approach (Figure 1d) to more specifically deliver radionuclides to tumor cells by first injecting BsPs that contain a tumor cell binding domain and an effector binding domain. Subsequently, radiolabeled effector molecules are introduced and interact with BsPs bound on the surface of tumor cells. Such an approach has been termed pretargeted radioimmunotherapy (PRIT) [47, 48]. Because the BsPs are non-radioactive and the radiolabeled effector molecules typically consist of modified small molecule metal chelators that can be rapidly cleared, PRIT 
can significantly improve the therapeutic index of radioisotope treatment compared to RIT [46, 49, 50], as well as increase the maximum tolerated dose for radionuclides [48]. Pagel et al. demonstrated that anti-CD45 PRIT improved the specificity of radiation delivery to leukemia in a rodent model, delivering twice as much radiation to bone marrow and five times more activity to the spleen than conventional RIT [51, 52]. In vivo PRIT was able to mediate broad tumor growth suppression and prolonged survival with the use of BsPs against receptors expressed at different levels on lymphoma cells, with CD20 and HLA-DR proving to be superior targets compared to CD22 [53, 54]. CD38-specific PRIT achieved tumor-to-blood ratios as high as 638:1 after 24 hours for a multiple myeloma model, compared to a ratio of $\sim 1: 1$ with conventional RIT [55]. Subbiah et al. reported that treating athymic mice bearing Ramos human Burkitt's lymphoma xenografts with a pretargeted system consisting of anti-CD20 scFvconjugated streptavidin (SA) and ${ }^{90}$ Y-DOTA-biotin cured $100 \%$ of mice with allowable toxicity, whereas conventional RIT with ${ }^{90} \mathrm{Y}-1 \mathrm{~F} 5$ at the same dose produced no cures, generated profound pancytopenia, and was lethal to all mice [56]. Zhang et al. demonstrated that both ${ }^{90}$ Y-DOTA-biotin and ${ }^{213}$ Bi-DOTA-biotin could both be used in combination with anti-CD25 scFv-conjugated SA for PRIT of a murine T-cell lymphoma xenograft model, with the beta-emitter ${ }^{90} \mathrm{Y}$ curing 10 of 10 mice and alpha-emitter ${ }^{213} \mathrm{Bi}$ curing 7 of 10 mice [57].

These encouraging results with PRIT studies in animal models led to clinical studies of PRIT, which have yielded promising results with reasonable tumor response rates and limited toxicity [58]. Forero et al. evaluated the pharmacokinetics and immunogenicity of an anti-CD20 scFv-SA conjugate in 15 patients with NHL [59]. Although the complete remission rate was low (2 of 15), the majority (12/15) patients exhibited no signs of hematologic toxicity, suggesting that the dose of radionuclide could be further increased. Another phase I/II PRIT clinical trial was performed using a chimeric anti-CD20 IgG-SA in combination with ${ }^{90}$ Y-DOTA-biotin. Six of seven NHL patients demonstrated significant tumor regression, with an estimated tumor-to-whole body dose ratio of 38:1. While six of the ten patients developed humoral responses to streptavidin, the transient nature of the responses appeared to result in no significant long-term effects $[60,61]$. Kraeber-Bodere et al. evaluated the therapeutic efficacy of PRIT using a bispecific monoclonal antibody that binds to carcinoembryonic antigen (CEA) and to a ${ }^{131}$ I-labeled effector molecule for PRIT of medullary thyroid cancer. Of the 17 patients treated, 4 reported pain relief, 5 demonstrated minor tumor responses, and 4 achieved biological responses (decrease in thyrocalcitonin); however, 9 patients also generated human anti-mouse antibodies [62-64]. 
While PRIT has led the way in preclinical and clinical studies of pretargeting, it is important to note that the applications for pretargeted strategies extend far beyond radiotherapy. For example, solid cancers, which will account for more than $90 \%$ of all newly diagnosed cancer cases and deaths in the United States in 2015 [65], are significantly more resistant to radioimmunotherapy compared to hematological malignancies such as NHL. To date, little is known about whether the pretargeting approach can enhance the delivery of other therapeutic agents such as nanoparticle drug carriers that can encapsulate and slowly release chemodrugs to solid tumors.

\section{Pretargeted drug delivery to heterogeneous tumors}

The growing interest in precision/personalized medicine, coupled with the incomplete treatment of heterogeneous cancers using common passively or single-ligand targeted therapies that can give rise to recurrent, more aggressive, and/or drug-resistant tumors [5, 29] highlights the need for alternative nanoparticle targeting strategies to improve treatment responses. The modular nature of pretargeted systems is particularly useful in addressing the challenge of and many barriers to effective drug delivery to heterogeneous tumors [32] because it enables pretargeted systems to be targeted to new or different tumor antigens by simply modifying the tumor binding domain of BsPs, as opposed to direct, ligand-based targeting systems that would require the formulation of a new nanoparticle system. This flexibility is expected to markedly reduce the production costs and complexity, as well as the potential regulatory burden, for pretargeted nanoparticles. Another equally appealing feature of pretargeting is the ability to pretarget multiple receptors simultaneously. The administration of a cocktail of pretargeting BsPs that can all bind to the same drug carrier could in theory enable the delivery of a drug carrier to the full spectrum of a patient's cancer cells (Figure 4). Drug cocktails containing mixtures of different MAbs have already been applied to cancer therapy, with one combination of pertuzumab, trastuzumab, and docetaxel significantly improving the overall survival of patients with HER2-positive breast cancer [66]. Antibody mixtures have also been used for in vivo imaging and diagnosis of tumors [67]. Additionally, pretargeting with individual or mixed BsPs was able to differentially label a range of human tumor cell lines in vitro (Figure 5a) [68, 69]. To our knowledge, no studies have been published on the simultaneous use of multiple pretargeting BsPs to enhance nanoparticle delivery to date, although Khaw et al. did report the receptor-dependent efficacy of doxorubicin nanoparticles pretargeted with anti-HER2 affibody-based BsPs in a dual tumor model [70]. In that study, tumor growth inhibition was achieved for HER2-positive BT-474 breast cancer tumors, while the HER2-negative BT-20 
breast cancer tumors were simultaneously unresponsive to the treatment, further emphasizing the opportunity for improved cancer treatment through appropriate targeting of all tumor cell populations.

1)

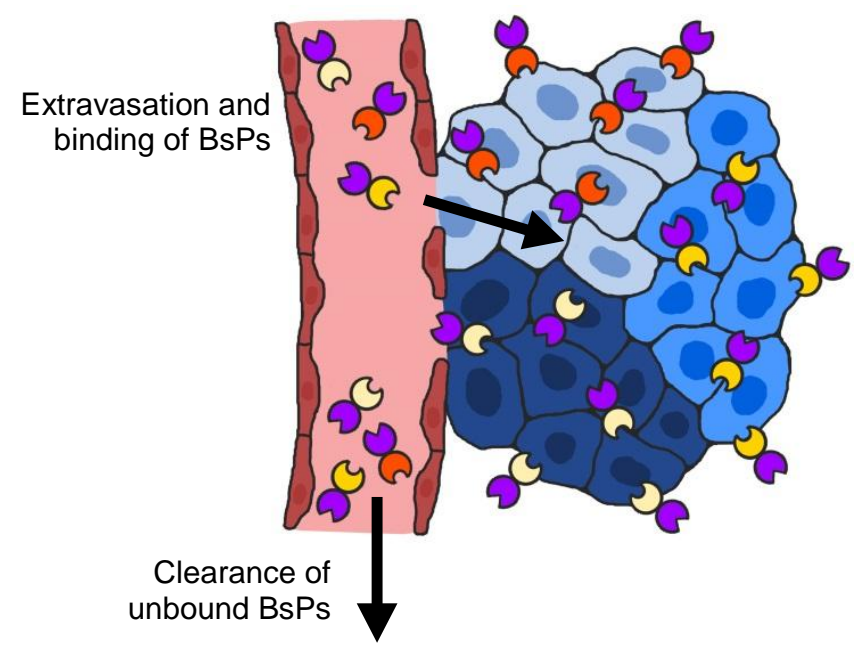

2)

Extravasation and binding of NPs

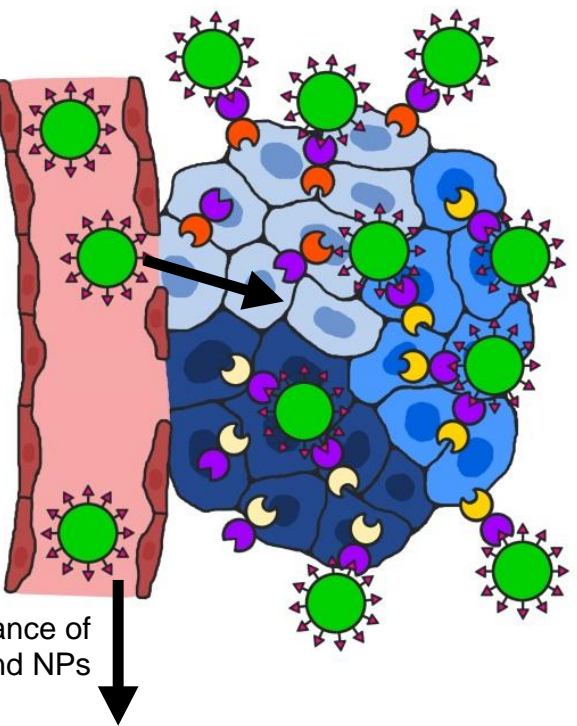

Figure 4. Pretargeted delivery of nanoparticles (NPs) to heterogeneous tumors. 1) A cocktail of bispecific proteins (BsPs) is administered and allowed to fully clear from systemic circulation prior to 2) dosing with nanoparticles that can be captured by BsPs on the tumor cell surface. To enable effective targeting of multiple tumor cell subpopulations using a single nanoparticle, the tumor antigen-binding domain (Figure 1e) of the BsPs can be modified to reflect the full diversity of tumor cells, while the effector (NP)-binding domain remains the same for all BsPs. 
a
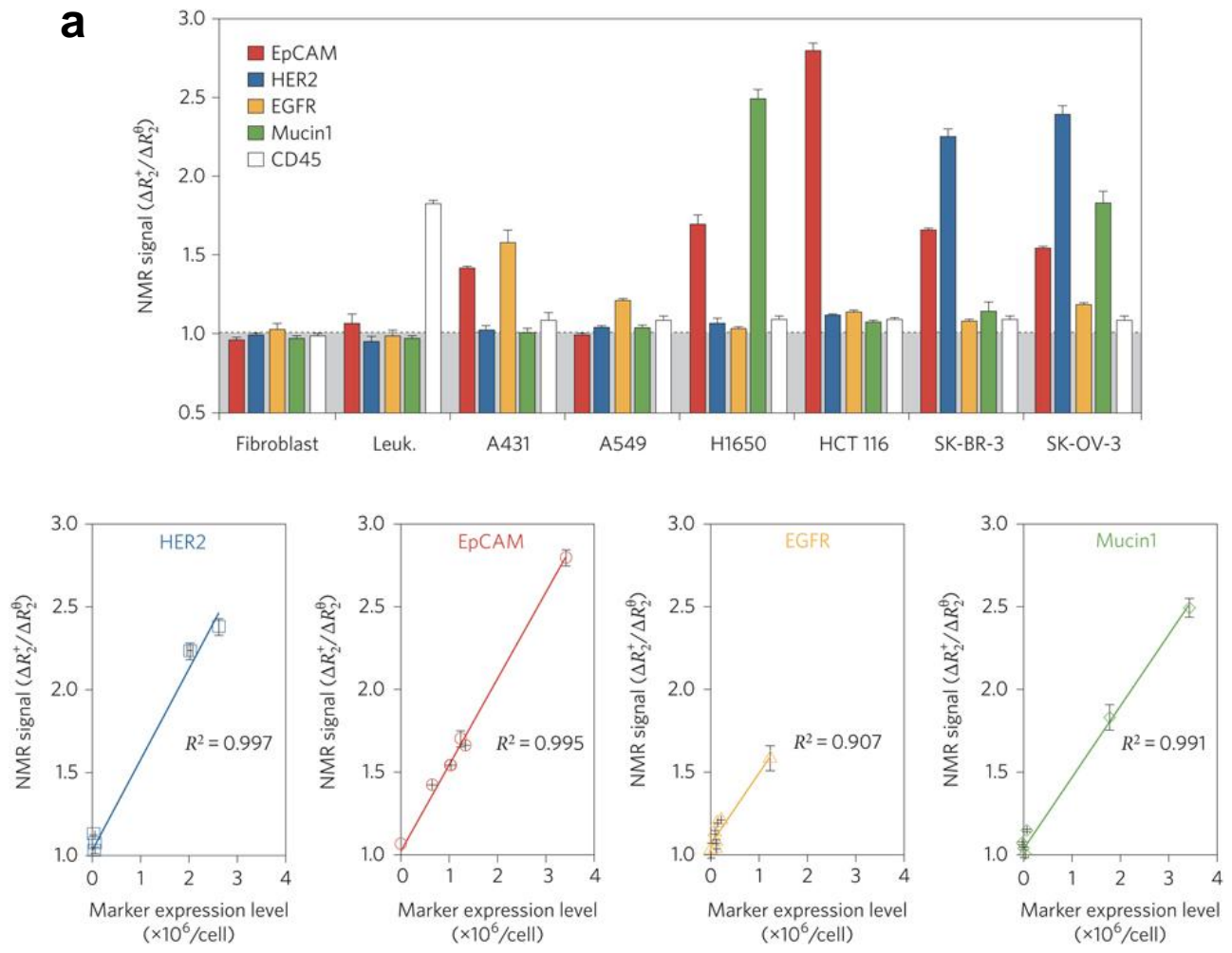

b
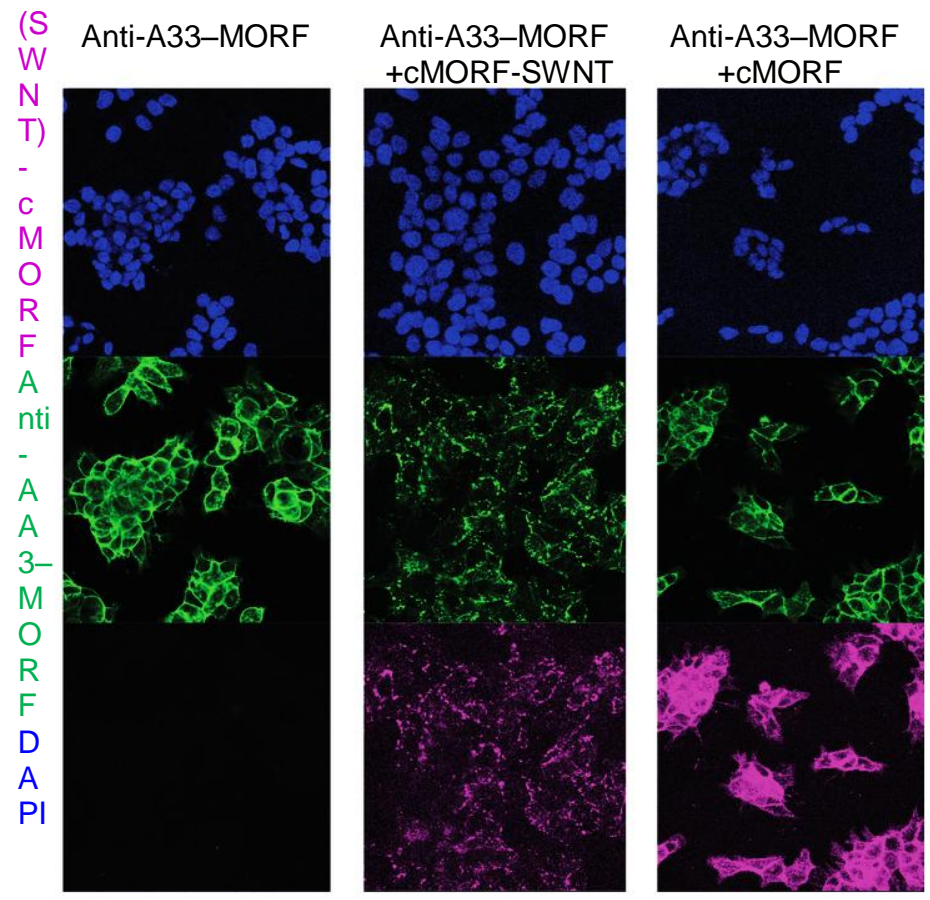

Figure 5. a) Diagnostic magnetic resonance profiling of human tumor cell lines, fibroblasts, and leukocytes using a pretargeted approach in vitro. The cells were labeled using various trans-cyclooctene (TCO)-conjugated antibodies followed by tetrazine-modified magneto-fluorescent nanoparticles (Tz-MFNPs) prior to the measurement of the transverse relaxation rate $\left(\mathrm{R}_{2}\right)$ [68]. b) Internalization of pretargeted single-walled carbon nanotubes (SWNTs). 
LS174T (A33-positive) colon carcinoma cells were preincubated anti-A33 antibodies conjugated to morpholino oligonucleotide (anti-A33-MORFs) for $4 \mathrm{~h}$ prior to washing and further incubation with complementary MORF (cMORF)-SWNT-AlexaFluor 647 or free cMORF-AlexaFluor 647. [71]

\section{Biological and pharmaceutical aspects and considerations of pretargeted drug delivery}

As multicomponent systems, the potential arsenal of pretargeted therapies is sizeable and highly diverse. Thus, many features (e.g., choice of target receptor/antigen, binding pair technology, and drug carrier) must be taken into account when developing a pretargeted drug delivery system to maximize transport of drug cargo to target cells and overall therapeutic efficacy. Because only a few publications have evaluated the use of pretargeting for nanoparticle delivery, the majority of the current knowledge about optimal pretargeted conditions have been gleaned from in vivo PRIT studies, but, due to the overlap of components for multistep targeting approaches (Figure 1d \& e), many of the lessons learned from PRIT likely apply to pretargeted nanocarriers.

\section{Binding pairs}

A key consideration of any pretargeted delivery approach is the binding interaction between the pretargeting BsP and nanoparticle effector, since the affinity of the binding pair directly influences the capture and retention of the drug carrier at the tumor site. In addition, the immunogenicity of the $\mathrm{BsP}$ and its interactions with endogenous ligands can also alter the efficacy of pretargeted therapies [45].

The first binding pairs used in pretargeted systems were based on antibody-hapten interactions. In 1985, Reardan et al. reported the development of antibodies against indium chelates of EDTA, and suggested the possibility of bispecific antibodies that can simultaneously recognize target antigens and metal chelates [46, 72]. Soon afterwards, Goodwin et al. developed an early pretargeted imaging approach through the injection of antichelate antibodies, followed by the administration of a radiolabel [73]. Since then, a number of antibodies against various haptens have been utilized for binding to effector molecules, including anti-DTPA complex [74, 75], antipeptide [76-78], anti-methotrexate [79], and anti-cotinine antibodies [80]. In addition to bispecific antibodies [75, $76,81]$, a range of antibody fragments and derivatives have been developed as pretargeting BsPs [45, 80, 82]. Most antibodies, including those used to capture radioisotope-carrying effector molecules in PRIT, exhibit nanomolar to high picomolar affinity $\left(\mathrm{K}_{\mathrm{D}} \sim 10^{-7}-10^{-10} \mathrm{M}\right)$ for the antigen target on the surface of cancer cells $[72,79,83]$. Since 
PRIT typically uses single radionuclide-loaded agents, the improvement of antibody-hapten binding through multivalency can significantly enhance the specificity of radioisotope localization and retention in tumor sites [76, 82, 83]. For example, the application of pretargeted bivalent haptens, termed the affinity enhancement system (AES), was able to improve the tumor biodistribution of bivalent ${ }^{111}$ In-diDTPA by more than 7 -fold compared to monovalent ${ }^{111}$ In-DTPA (tumor biodistribution: $52.9 \%$ vs. $7.6 \% \mathrm{ID} / \mathrm{g}$ at $1 \mathrm{~h}$ and $92.5 \% \mathrm{vs.} 0.9 \% \mathrm{ID} / \mathrm{g}$ at $72 \mathrm{~h}$, respectively) [84].

As a binding pair with one of the strongest noncovalent binding affinities $\left(\mathrm{K}_{\mathrm{D}} \sim 10^{-14}-10^{-15} \mathrm{M}\right)$, the streptavidin (SA)-biotin system was quickly adopted by the pretargeting field [85, 86]. Additionally, SA is a tetravalent protein and could enable the capture of multiple biotinylated drug molecules. SA and biotin can be attached to tumor-specific pretargeting proteins and/or effector molecules through a variety of methods, including direct conjugation [56, 87, 88], genetic engineering of fusion proteins [89-91], and enzymatic conjugation [92]. While SA-based PRIT systems have demonstrated increased tumor specificity and higher therapeutic indices relative to directly targeted systems $[56,91]$ and have even been evaluated in clinical trials $[59,88,93]$, the immunogenic nature of SA, a bacterial protein, represents a major challenge to widespread clinical use of SA-biotin binding pairs $[59,61]$. The immunogenicity of SA can be reduced through site-specific mutations $[94,95]$, although it remains unclear whether these SA mutants will be sufficiently hypoimmunogenic to allow for repeated dosing in humans. The problem of interference from endogenous biotin [96], which necessitates the use of biotin-free feed for in vivo studies, could also be potentially addressed by SA mutants that selectively bind bis-biotin instead of biotin $[97,98]$. Other proteins that naturally bind to specific substrates (e.g., enzymes) can also be modified to bind to exogenous molecules for use in pretargeting, although only a few such systems have been reported in the literature $[99,100]$.

The majority of published pretargeted and multistep targeting systems utilize antibody-hapten or proteinligand interactions, but research in areas such as complementary synthetic nucleic acids and peptides and bioorthogonal chemistry continues to generate novel classes of binding pairs. Morpholinos (MORFs) are the most popular class of synthetic nucleic acid analogs for pretargeting using complementary nucleic acids and have been evaluated preclinically in combination with tumor-specific pretargeting antibodies and a variety of radionuclides [101-105]. In addition to relatively low immunogenicity, optimized complementary morpholinos exhibit high 
specificity and binding affinity [106], and the use of bivalent MORFs may further enhance affinity [107].

Bioorthogonal chemistry comprises reactions that can rapidly occur in a living system with high selectivity and without any off-target reactions or toxicity. These properties enable pretargeting using small molecule binding pairs with low immunogenicity, although the relative merits of different bioorthogonal chemistries vary based on reaction kinetics, complexity of synthesis, and stability of the resulting conjugate (see refs $[45,108,109])$. Rossin et al. demonstrated the feasibility of using "click" chemistry for pretargeting of radioisotopes in vivo by treating tumor-bearing mice with an anti-TAG72 antibody (CC49) modified with trans-cyclooctene (TCO), which then reacted with ${ }^{111}$ In-tetrazine $\left({ }^{111} \mathrm{In}-\mathrm{Tz}\right)$ administered $24 \mathrm{~h}$ later [110]. The CC49-TCO predosed mice exhibited a tumor uptake of $4.2 \% \mathrm{ID} / \mathrm{g}$ and tumor-to-muscle (T/M) ratio of 13.1, compared to tumor uptake and T/M ratios of $0.3 \% \mathrm{ID} / \mathrm{g}$ and 0.5 and $1 \% \mathrm{ID} / \mathrm{g}$ and 2.1 for unmodified CC49 and control Ab-TCO groups, respectively. Further preclinical studies have confirmed the utility of TCO-tetrazine and other bioorthogonal chemistries for tumor imaging and treatment $[45,111-113]$.

While all of the aforementioned classes of binding pairs have also been used in the pretargeting of nanoparticles and other potential drug carriers [68, 114-116], the ability of pretargeted systems to actually deliver therapeutics to tumor cells has only been evaluated in a few studies [70, 117-119]. Pretargeted poly-lysine polymers [120], liposomes [121], and carbon nanotubes [71] have been used to deliver higher doses of encapsulated or conjugated radionuclides to both solid tumor and hematologic cancer cells, suggesting that the application of nanocarriers could further improve the efficacy of PRIT. In the context of cancer chemotherapy, pretargeted biotinylated polymeric nanoparticles loaded with pactilaxel (PTX) increased the in vitro cell killing of glioma and breast cancer cells, relative to free drug or Taxol and nontargeted nanoparticles $[117,118]$. The injection of an antiHER2 affibody-anti-DTPA Fab complex (BAAC) $8 \mathrm{~h}$ prior to the administration of ${ }^{99 \mathrm{~m}}$ Tc-DTPA-succinylated polylysine enabled the specific labeling of tumors $(5.3 \% \mathrm{ID} / \mathrm{g}$ vs. $0.5 \% \mathrm{ID} / \mathrm{g}$ for anti-DTPA Fab-pretargeted particles) [70]. BAAC pretargeting of doxorubicin- and DTPA-conjugated polyglutamic acid produced tumor growth inhibition results that were similar to those of free doxorubicin, but pretargeting through the combination of BAAC and polymer-drug conjugate minimized weight loss in mice relative to the free drug treatment [70], underscoring the ability of pretargeting to improve the therapeutic index of chemotherapeutics in vivo. 
Although BsP considerations such as immunogenicity and competition with endogenous ligands apply to both PRIT and pretargeted nanoparticles, other features and characteristics of binding pairs required for pretargeted drug delivery systems may differ from those for pretargeting based on small molecule effectors. Nanoparticles are inherently highly multivalent due to their large surface area, which allows the grafting of tens to possibly thousands of a given binding partner moiety. Thus, BsPs with lower affinity to a hapten may still be able to capture haptencoated nanoparticles with high avidity compared to individual radiolabeled haptens. However, as is the case with actively targeted systems, the incorporation of peptides, nucleic acids, proteins and other macromolecular components onto drug carrier particles could negatively impact their circulation kinetics and efficiency of extravasation into tumors. For binding pairs that consist of a large protein and a smaller moiety (e.g., SA-biotin, antibody-hapten), the smaller, the more immunologically inert moiety should be assigned to the effector nanoparticle to minimize MPS clearance. Steric considerations may further support the modification of drug carriers with smaller BsP-binding components. For instance, Haun et al. reported that, in addition to relatively to providing a 10- to 15-fold increase in cell binding relative to directly targeted iron oxide nanoparticles, a pretargeted antibody-TCO/Tz-NP system demonstrated significantly higher fluorescent labeling of various tumor cell lines, compared to an antibody-biotin/avidin-NP system [68]. The authors attributed this difference to the large footprint of avidin $(\sim 67 \mathrm{kDa})$ on the particles, which likely resulted in the reduced accessibility and valency of biotin-binding sites. The use of a PEG spacer for TCO-antibody modificiation also improved the pretargeting of quantum dots by reducing masking of reactive groups [122].

\section{Target antigen $(s)$}

A diverse array of receptors and other antigens overexpressed on tumor cells have been exploited for active targeting of nanoparticles and for RIT [1, 123]. In contrast, the number of target cancer antigens/receptors suitable for pretargeted approaches is certainly more limited. The multistep nature of pretargeting requires that the tumor cell-binding BsP must remain on the tumor cell surface to capture subsequently injected effector drug carriers. Indeed, the majority of PRIT studies to date utilize BsPs that target epitopes generally considered to be noninternalizing, including CD20, CD45, TAG72, and CEA [49, 59, 71, 89, 107, 116, 119]. However, Liu et al. observed the fairly rapid internalization of radiolabeled anti-TAG72 and anti-CEA antibodies, with about $60 \%$ of the antibodies internalized by LS174T colon carcinoma cells after $5 \mathrm{~h}$ [102]. Similarly, although HER2 is thought 
to be an internalizing epitope, pretargeting using bispecific antibodies against HER2 mediated enhanced tumor accumulation in vivo [70,80,124]. Whether these apparently counterintuitive results are due to differences in antibody internalization kinetics between in vitro and in vivo conditions (e.g., differences in receptor density, receptor turnover rates, and/or endocytosis and cell signalling pathways), dosing of the pretargeting molecules at sufficiently high levels that compensate for loss due to antigen/BsP internalization, or other factors remains unknown.

While the pretargeting molecule should initially remain non-internalized, many therapeutics require intracellular delivery to be effective and/or exhibit maximal potency; thus, the ideal pretargeted nanoparticle must be internalized only after binding of the drug carrier (Figure 1). Although internalization mediated by a noninternalizing pretargeting molecule may appear paradoxical, cellular entry could be achieved by relying on the eventual endocytosis of bound receptors or, more preferably, through multivalent nanoparticle binding effects such as crosslinking of receptors. Mulvey et al. observed that anti-A33-MORF conjugates remained stably on the surface of LS174T cells for up to $24 \mathrm{~h}$, and that the addition of complementary MORF-modified carbon nanotubes resulted intracellular punctate staining indicative of internalization (Figure 5b) [71]. In contrast, free complementary MORFs failed to induce internalization (Figure 5b). Gunn et al. similarly reported that iron oxide nanoparticles pretargeted to CD20-expressing cells were found in endosomes, as visualized by transmission electron microscopy [114]. These results suggest that BsPs that bind non-internalizing epitopes can still facilitate pretargeted intracellular delivery of nanocarriers.

\section{Pharmacokinetics and biodistribution}

The theoretical improvements in the therapeutic index of drug delivery systems that can be achieved using pretargeting are based on the decoupling of the tumor targeting vs. drug-carrying functions. This in turn implies that the efficacy of a given pretargeted system is dependent on the pharmacokinetics and biodistribution of each component. One of the most important requirements is that the pretargeting BsPs are maximally cleared from systemic circulation prior to the administration of the drug carrier, particularly for pretargeted systems based on high affinity binding pairs such as SA-biotin. Indeed, SA-coated liposomes were detectable in circulation for at least $24 \mathrm{~h}$ after i.v. administration in mice, whereas SA-coated liposomes premixed with biotinylated anti-Thy 1.2 antibodies prior to dosing were rapidly cleared within $4 \mathrm{~h}$ [119], illustrating the potential problem of circulating 
BsP binding to effector nanoparticles before the particles can extravasate into the tumor. Correspondingly, Karacay et al. found that, while an anti-CEA IgG $\mathrm{x}$ anti-DTPA Fab' conjugate demonstrated superior tumor labeling relative to $\left.\mathrm{F}(\mathrm{ab})_{2}\right)_{2} \mathrm{Fab}$ ' and Fab' $\mathrm{x}$ Fab' constructs, the $\mathrm{F}\left(\mathrm{ab}{ }^{\prime}\right)_{2} \times \mathrm{Fab}$ ' conjugate provided better pretargeting of a divalent DTPA peptide due to the high residual blood concentration of IgG x Fab' even 6 days after administration [74]. In order to simultaneously optimize tumor distribution and retention along with systemic clearance, a variety of techniques have been used to modify the size, valency, and composition of pretargeting BsPs, including the "dockand-lock" method $[125,126]$ and fusion protein engineering $[59,89,99]$.

An alternative approach to ensure elimination of residual pretargeting molecules from the systemic circulation is the use of clearing agents (CAs) prior to the dosing of nanoparticles or therapeutic effector molecules. These multivalent agents are generally designed to bind tightly to the pretargeting molecules and are sufficiently large enough to be rapidly cleared from the systemic circulation without extravasating into tumors. Previously reported CAs include secondary antibodies [127] and avidin [128], as well as biotinylated and galactosylated human serum albumin [129] and dendrimers $[52,89]$. The use of a CA can effectively purge circulating BsP molecules (reducing blood concentrations by up to 10 -fold) without affecting the tumor accumulation of pretargeting molecules $[89,128,130]$. The potential drawback of CA use is the addition of yet another dose and wait step to the course of therapy. For example, the use of CAs with the sequential combination of biotinylated antibodies, SA, and finally biotinylated radionuclide resulted in a 5-step PRIT strategy (biotinylated MAb/avidin $\mathrm{CA} /$ streptavidin/biotinylated CA/biotinylated radiolabeled chelate) $[131,132]$ Although the radioimmunotherapy was well-tolerated and effective in glioma patients, with a median survival of 33.5 months (compared to 8 months for untreated control patients) in a nonrandomized phase I/II study, the need for several parenteral injections to deliver a single dose of radiation or drug not only introduces a high degree of complexity but also increases the cost of therapy. A simpler 2-step approach is likely far more preferable, particularly when using antibody-hapten binding pairs. These pretargeted systems appear to better tolerate the presence of minute amounts of uncleared pretargeting BsP, possibly due to the lower affinity and the dissociation of BsP-effector complexes formed in the blood [133].

The pharmacokinetics of the pretargeted drug carrier must also be taken into consideration. Because the commonly utilized pretargeting molecules are generally much smaller than nanoparticle drug carriers, the overall 
tumor distribution and accumulation of pretargeted systems is therefore limited by the circulation and extravasation kinetics of the drug carrier. To minimize premature elimination from the circulation and maximize tumor accumulation, drug carriers should be effectively coated with stealth polymers, whereas the use of of bulky, charged, and/or hydrophobic moieties to facilitate particle binding to the pretargeting molecule should be avoided if possible.

\section{Challenges and unknowns}

The combination of a bispecific pretargeting cocktail with nanoparticle drug carriers is a promising but vastly underexplored approach to targeting nanoparticles to heterogeneous tumors. Thus, many aspects of this proposed strategy must be rigorously evaluated to confirm its suitability for clinical applications.

One of the major challenges is that a greater dose of pretargeting BsP could potentially reduce nanoparticle binding and accumulation to tumor cells. Because tumor receptor expression varies both spatially and temporally, and receptor testing is typically performed on primary tumor biopsies obtained close to the time of diagnosis, "personalized" pretargeting cocktails based on those patient biopsy results is unlikely to capture the full heterogeneity of cancer cells in a patient over time, particularly for relapsed and/or highly metastatic tumors [4]. Thus, truly personalized pretargeted therapy would greatly benefit from improvements in noninvasive molecular profiling of cancers $[29,134]$. As an alternative to the fine-tuning of individual pretargeting cocktails, the properties of BsPs could be optimized to allow rapid elmination of non-binding BsPs from the circulation. The mechanism, rate, and extent of pretargeting BsP clearance with and without the use of clearing agents must be carefully investigated, particularly since Pagel and colleagues observed that the administration of high doses of MAb-SA conjugates specific to receptors poorly expressed on certain lymphoma tumors overloaded the capacity of mice to hepatically clear MAb-SA/CA complexes, resulting in low tumor-to-normal organ biodistribution ratios and toxicity $[53,135]$. The increased doses of total protein required for a cocktail pretargeting approach may also affect the immunogenicity of the pretargeting BsPs used.

Additionally, the limited number of appropriate target receptor/antibody combinations that have been evaluated for pretargeting to date may hinder the development of useful pretargeting cocktails. The main driving forces behind the discovery of novel tumor-specific receptors and their corresponding ligands/antibodies are diagnostic biomarkers, imaging applications and targeted drug and MAb therapy. Unfortunately, few of these 
studies focus on non-internalizing antibodies, a critical requirement of pretargeting. However, the use of (pre)clincially validated ligands and therapeutic MAbs could lead to fortuitous combinations for pretargeting. For example, anti-CD20 MAbs can induce apoptosis clinically [136], and anti-CD20 Fab' fragments linked to MORFs have been found to also induce apoptosis of B-cell lymphomas in vitro and inhibit development of diffuse tumors in vivo upon crosslinking by cMORF-modified polymers [115]. The use of antibodies with inherent therapeutic efficacy for pretargeting of drug carriers could allow for synergistic treatment effects. Improvements in the generation of diverse bispecific proteins and antibodies will also certainly expand the diversity of available pretargeting molecules $[125,126,137]$.

Other concerns regarding the application of pretargeted drug delivery systems include the clinical feasiblity of multistep parenteral injections and the poor tumor accumulation of many drug carriers in patient tumors. Similar to passively and actively targeted nanoparticles, the tumor accumulation of pretargeted drug carriers would still rely on the EPR effect [119], which has been found to be highly variable [19].

\section{Conclusion}

Despite marked advances in biotechnology, nanotechnology and drug delivery, effective therapy for cancer remains exceedingly challenging, with few treatment options that can provide durable suppression or elimination of the tumor without resulting in eventual recurrence and/or the development of drug-resistant tumors. Emerging insights into tumor physiology have underscored tumor heterogeneity as one of the key bottlenecks to targeted therapy. The concept of pretargeting using a cocktail of bispecific pretargeting proteins combines the strengths of precision medicine and personalized medicine by offering the potential to deliver nanoparticle therapeutics to diverse cell populations while avoiding the pharmacokinetic pitfalls typically associated with actively targeted nanoparticles. Although the radioimmunotherapy field has offered substantial evidence supporting the pretargeting strategy, its application for enhancing targeted delivery of nanoparticle therapeutics remains underexplored to date. We believe further rigorous evaluation of pretargeted NP systems is both warranted and needed to confirm whether pretargeting can indeed prove superior to current passive and active targeting approaches. 


\section{Acknowledgements}

Financial support was provided by the PhRMA Foundation Predoctoral Fellowship (Q.Y.), the National Science Foundation Graduate Research Fellowship Program (C.L.P., DGE-1144081), The David and Lucile Packard Foundation (2013-39274, S.K.L.), National Institutes of Health (R21EB017938, S.K.L.), UNC Research

Opportunities Initiative grant in Pharmacoengineering, Carolina Center for Cancer Nanotechnology Excellence (U54CA151652) Pilot Grants Program and startup funds from the Eshelman School of Pharmacy and Lineberger Comprehensive Cancer Center (S.K.L.).

\section{References}

[1] N. Bertrand, J. Wu, X. Xu, N. Kamaly, O.C. Farokhzad, Cancer nanotechnology: the impact of passive and active targeting in the era of modern cancer biology, Adv Drug Deliv Rev, 66 (2014) 2-25.

[2] Y.H. Bae, K. Park, Targeted drug delivery to tumors: Myths, reality and possibility, J Control Release, 153 (2011) 198-205.

[3] V.J. Venditto, F.C. Szoka Jr, Cancer nanomedicines: So many papers and so few drugs!, Advanced Drug Delivery Reviews, 65 (2013) 80-88.

[4] F. Janku, Tumor heterogeneity in the clinic: is it a real problem?, Therapeutic Advances in Medical Oncology, 6 (2014) 43-51.

[5] P.L. Bedard, A.R. Hansen, M.J. Ratain, L.L. Siu, Tumour heterogeneity in the clinic, Nature, 501 (2013) 355364.

[6] A.M. Gonzalez-Angulo, F. Morales-Vasquez, G.N. Hortobagyi, Overview of resistance to systemic therapy in patients with breast cancer, Adv Exp Med Biol, 608 (2007) 1-22.

[7] Y. Matsumura, H. Maeda, A new concept for macromolecular therapeutics in cancer chemotherapy: mechanism of tumoritropic accumulation of proteins and the antitumor agent smancs, Cancer Res, 46 (1986) 6387-6392.

[8] J. Fang, H. Nakamura, H. Maeda, The EPR effect: Unique features of tumor blood vessels for drug delivery, factors involved, and limitations and augmentation of the effect, Adv Drug Deliv Rev, 63 (2011) 136-151.

[9] H. Kobayashi, R. Watanabe, P.L. Choyke, Improving conventional enhanced permeability and retention (EPR) effects; what is the appropriate target?, Theranostics, 4 (2013) 81-89.

[10] V. Torchilin, Tumor delivery of macromolecular drugs based on the EPR effect, Adv Drug Deliv Rev, 63 (2011) 131-135.

[11] R. Toy, P.M. Peiris, K.B. Ghaghada, E. Karathanasis, Shaping cancer nanomedicine: the effect of particle shape on the in vivo journey of nanoparticles, Nanomedicine (Lond), 9 (2014) 121-134.

[12] D. Banerjee, R. Harfouche, S. Sengupta, Nanotechnology-mediated targeting of tumor angiogenesis, Vasc Cell, 3 (2011) 3.

[13] M.A. Phillips, M.L. Gran, N.A. Peppas, Targeted Nanodelivery of Drugs and Diagnostics, Nano Today, 5 (2010) 143-159.

[14] V.P. Torchilin, Passive and active drug targeting: drug delivery to tumors as an example, Handb Exp Pharmacol, (2010) 3-53.

[15] S. Salmaso, P. Caliceti, Stealth properties to improve therapeutic efficacy of drug nanocarriers, J Drug Deliv, 2013 (2013) 374252.

[16] Z. Amoozgar, Y. Yeo, Recent advances in stealth coating of nanoparticle drug delivery systems, Wiley Interdiscip Rev Nanomed Nanobiotechnol, 4 (2012) 219-233.

[17] J. Kopecek, P. Kopeckova, HPMA copolymers: origins, early developments, present, and future, Adv Drug Deliv Rev, 62 (2010) 122-149. 
[18] H. Maeda, Toward a full understanding of the EPR effect in primary and metastatic tumors as well as issues related to its heterogeneity, Adv Drug Deliv Rev, (2015).

[19] E. Huynh, G. Zheng, Cancer nanomedicine: addressing the dark side of the enhanced permeability and retention effect, Nanomedicine (Lond), (2015) 1-3.

[20] U. Prabhakar, H. Maeda, R.K. Jain, E.M. Sevick-Muraca, W. Zamboni, O.C. Farokhzad, S.T. Barry, A. Gabizon, P. Grodzinski, D.C. Blakey, Challenges and key considerations of the enhanced permeability and retention effect for nanomedicine drug delivery in oncology, Cancer Res, 73 (2013) 2412-2417.

[21] R. Bazak, M. Houri, S. El Achy, S. Kamel, T. Refaat, Cancer active targeting by nanoparticles: a comprehensive review of literature, J Cancer Res Clin Oncol, 141 (2015) 769-784.

[22] T.M. Allen, Ligand-targeted therapeutics in anticancer therapy, Nat Rev Cancer, 2 (2002) 750-763.

[23] J.R. Beech, S.J. Shin, J.A. Smith, K.A. Kelly, Mechanisms for targeted delivery of nanoparticles in cancer, Curr Pharm Des, 19 (2013) 6560-6574.

[24] Z. Cheng, A. Al Zaki, J.Z. Hui, V.R. Muzykantov, A. Tsourkas, Multifunctional nanoparticles: cost versus benefit of adding targeting and imaging capabilities, Science, 338 (2012) 903-910.

[25] F. Gu, L. Zhang, B.A. Teply, N. Mann, A. Wang, A.F. Radovic-Moreno, R. Langer, O.C. Farokhzad, Precise engineering of targeted nanoparticles by using self-assembled biointegrated block copolymers, Proc Natl Acad Sci U S A, 105 (2008) 2586-2591.

[26] D.R. Elias, A. Poloukhtine, V. Popik, A. Tsourkas, Effect of ligand density, receptor density, and nanoparticle size on cell targeting, Nanomedicine (Lond), 9 (2013) 194-201.

[27] A. Fakhari, A. Baoum, T.J. Siahaan, K.B. Le, C. Berkland, Controlling ligand surface density optimizes nanoparticle binding to ICAM-1, J Pharm Sci, 100 (2011) 1045-1056.

[28] E. Moradi, Vllasaliu, D., Garnett, M., Falcone, F., \& Stolnik, S, Ligand density and clustering effects on endocytosis of folate modified nanoparticles, RSC Advances, 2 (2012) 3025-3033.

[29] M. Jamal-Hanjani, S.A. Quezada, J. Larkin, C. Swanton, Translational implications of tumor heterogeneity, Clin Cancer Res, 21 (2015) 1258-1266.

[30] H.J. Lee, A.N. Seo, E.J. Kim, M.H. Jang, K.J. Suh, H.S. Ryu, Y.J. Kim, J.H. Kim, S.A. Im, G. Gong, K.H. Jung, I.A. Park, S.Y. Park, HER2 heterogeneity affects trastuzumab responses and survival in patients with HER2positive metastatic breast cancer, Am J Clin Pathol, 142 (2014) 755-766.

[31] R.F. Schwarz, C.K. Ng, S.L. Cooke, S. Newman, J. Temple, A.M. Piskorz, D. Gale, K. Sayal, M. Murtaza, P.J. Baldwin, N. Rosenfeld, H.M. Earl, E. Sala, M. Jimenez-Linan, C.A. Parkinson, F. Markowetz, J.D. Brenton, Spatial and temporal heterogeneity in high-grade serous ovarian cancer: a phylogenetic analysis, PLoS Med, 12 (2015) e1001789.

[32] T.A. Denison, and You Han Bae. Cancer Targeted Drug Delivery., Heterogeneity of Cancers and Its Implication for Targeted Drug Delivery, Springer, (2013) 337-362.

[33] L. Ding, M.J. Ellis, S. Li, D.E. Larson, K. Chen, J.W. Wallis, C.C. Harris, M.D. McLellan, R.S. Fulton, L.L. Fulton, R.M. Abbott, J. Hoog, D.J. Dooling, D.C. Koboldt, H. Schmidt, J. Kalicki, Q. Zhang, L. Chen, L. Lin, M.C. Wendl, J.F. McMichael, V.J. Magrini, L. Cook, S.D. McGrath, T.L. Vickery, E. Appelbaum, K. Deschryver, S. Davies, T. Guintoli, L. Lin, R. Crowder, Y. Tao, J.E. Snider, S.M. Smith, A.F. Dukes, G.E. Sanderson, C.S. Pohl, K.D. Delehaunty, C.C. Fronick, K.A. Pape, J.S. Reed, J.S. Robinson, J.S. Hodges, W. Schierding, N.D. Dees, D. Shen, D.P. Locke, M.E. Wiechert, J.M. Eldred, J.B. Peck, B.J. Oberkfell, J.T. Lolofie, F. Du, A.E. Hawkins, M.D. O'Laughlin, K.E. Bernard, M. Cunningham, G. Elliott, M.D. Mason, D.M. Thompson, Jr., J.L. Ivanovich, P.J. Goodfellow, C.M. Perou, G.M. Weinstock, R. Aft, M. Watson, T.J. Ley, R.K. Wilson, E.R. Mardis, Genome remodelling in a basal-like breast cancer metastasis and xenograft, Nature, 464 (2010) 999-1005.

[34] A.P. Albino, K.O. Lloyd, A.N. Houghton, H.F. Oettgen, L.J. Old, Heterogeneity in surface antigen and glycoprotein expression of cell lines derived from different melanoma metastases of the same patient. Implications for the study of tumor antigens, J Exp Med, 154 (1981) 1764-1778.

[35] M. Gerlinger, A.J. Rowan, S. Horswell, J. Larkin, D. Endesfelder, E. Gronroos, P. Martinez, N. Matthews, A. Stewart, P. Tarpey, I. Varela, B. Phillimore, S. Begum, N.Q. McDonald, A. Butler, D. Jones, K. Raine, C. Latimer, C.R. Santos, M. Nohadani, A.C. Eklund, B. Spencer-Dene, G. Clark, L. Pickering, G. Stamp, M. Gore, Z. Szallasi, J. Downward, P.A. Futreal, C. Swanton, Intratumor heterogeneity and branched evolution revealed by multiregion sequencing, N Engl J Med, 366 (2012) 883-892.

[36] C. Arslan, E. Sari, S. Aksoy, K. Altundag, Variation in hormone receptor and HER-2 status between primary and metastatic breast cancer: review of the literature, Expert Opin Ther Targets, 15 (2011) 21-30.

[37] C.E. Meacham, S.J. Morrison, Tumour heterogeneity and cancer cell plasticity, Nature, 501 (2013) 328-337. 
[38] M.R. Junttila, F.J. de Sauvage, Influence of tumour micro-environment heterogeneity on therapeutic response, Nature, 501 (2013) 346-354.

[39] J.N. Jakobsen, J.B. Sorensen, Intratumor heterogeneity and chemotherapy-induced changes in EGFR status in non-small cell lung cancer, Cancer Chemother Pharmacol, 69 (2012) 289-299.

[40] Y.P. Choi, H.S. Shim, M.Q. Gao, S. Kang, N.H. Cho, Molecular portraits of intratumoral heterogeneity in human ovarian cancer, Cancer Lett, 307 (2011) 62-71.

[41] B. Vogelstein, N. Papadopoulos, V.E. Velculescu, S. Zhou, L.A. Diaz, Jr., K.W. Kinzler, Cancer genome landscapes, Science, 339 (2013) 1546-1558.

[42] J. Liu, S.K. Lau, V.A. Varma, R.A. Moffitt, M. Caldwell, T. Liu, A.N. Young, J.A. Petros, A.O. Osunkoya, T. Krogstad, B. Leyland-Jones, M.D. Wang, S. Nie, Molecular mapping of tumor heterogeneity on clinical tissue specimens with multiplexed quantum dots, ACS Nano, 4 (2010) 2755-2765.

[43] Z.Z. Yang, D.M. Grote, S.C. Ziesmer, B. Xiu, A.J. Novak, S.M. Ansell, PD-1 expression defines two distinct T-cell sub-populations in follicular lymphoma that differentially impact patient survival, Blood Cancer J, 5 (2015) e281.

[44] T.A. Yap, M. Gerlinger, P.A. Futreal, L. Pusztai, C. Swanton, Intratumor heterogeneity: seeing the wood for the trees, Sci Transl Med, 4 (2012) 127ps110.

[45] F.C. van de Watering, M. Rijpkema, M. Robillard, W.J. Oyen, O.C. Boerman, Pretargeted imaging and radioimmunotherapy of cancer using antibodies and bioorthogonal chemistry, Front Med (Lausanne), 1 (2014) 44. [46] R.B. Walter, O.W. Press, J.M. Pagel, Pretargeted radioimmunotherapy for hematologic and other malignancies, Cancer Biother Radiopharm, 25 (2010) 125-142.

[47] D.M. Goldenberg, C.H. Chang, E.A. Rossi, W. J, McBride, R.M. Sharkey, Pretargeted molecular imaging and radioimmunotherapy, Theranostics, 2 (2012) 523-540.

[48] D.J. Green, J.M. Pagel, A. Pantelias, N. Hedin, Y. Lin, D.S. Wilbur, A. Gopal, D.K. Hamlin, O.W. Press, Pretargeted radioimmunotherapy for B-cell lymphomas, Clin Cancer Res, 13 (2007) 5598s-5603s.

[49] J.M. Pagel, N. Hedin, K. Subbiah, D. Meyer, R. Mallet, D. Axworthy, L.J. Theodore, D.S. Wilbur, D.C. Matthews, O.W. Press, Comparison of anti-CD20 and anti-CD45 antibodies for conventional and pretargeted radioimmunotherapy of B-cell lymphomas, Blood, 101 (2003) 2340-2348.

[50] D.J. Green, J.M. Pagel, E.R. Nemecek, Y. Lin, A. Kenoyer, A. Pantelias, D.K. Hamlin, D.S. Wilbur, D.R. Fisher, J.G. Rajendran, A.K. Gopal, S.I. Park, O.W. Press, Pretargeting CD45 enhances the selective delivery of radiation to hematolymphoid tissues in nonhuman primates, Blood, 114 (2009) 1226-1235.

[51] J.M. Pagel, D.C. Matthews, A. Kenoyer, D.K. Hamlin, D.S. Wilbur, D.R. Fisher, A.K. Gopal, Y. Lin, L. Saganic, F.R. Appelbaum, O.W. Press, Pretargeted radioimmunotherapy using anti-CD45 monoclonal antibodies to deliver radiation to murine hematolymphoid tissues and human myeloid leukemia, Cancer Res, 69 (2009) 185-192. [52] J.M. Pagel, A.L. Kenoyer, T. Back, D.K. Hamlin, D.S. Wilbur, D.R. Fisher, S.I. Park, S. Frayo, A. Axtman, N. Orgun, J. Orozco, J. Shenoi, Y. Lin, A.K. Gopal, D.J. Green, F.R. Appelbaum, O.W. Press, Anti-CD45 pretargeted radioimmunotherapy using bismuth-213: high rates of complete remission and long-term survival in a mouse myeloid leukemia xenograft model, Blood, 118 (2011) 703-711.

[53] J.M. Pagel, N. Orgun, D.K. Hamlin, D.S. Wilbur, T.A. Gooley, A.K. Gopal, S.I. Park, D.J. Green, Y. Lin, O.W. Press, A comparative analysis of conventional and pretargeted radioimmunotherapy of B-cell lymphomas by targeting CD20, CD22, and HLA-DR singly and in combinations, Blood, 113 (2009) 4903-4913.

[54] J.M. Pagel, A. Pantelias, N. Hedin, S. Wilbur, L. Saganic, Y. Lin, D. Axworthy, D.K. Hamlin, D.S. Wilbur, A.K. Gopal, O.W. Press, Evaluation of CD20, CD22, and HLA-DR targeting for radioimmunotherapy of B-cell lymphomas, Cancer Res, 67 (2007) 5921-5928.

[55] D.J. Green, N.N. Orgun, J.C. Jones, M.D. Hylarides, J.M. Pagel, D.K. Hamlin, D.S. Wilbur, Y. Lin, D.R. Fisher, A.L. Kenoyer, S.L. Frayo, A.K. Gopal, J.J. Orozco, T.A. Gooley, B.L. Wood, W.I. Bensinger, O.W. Press, A preclinical model of CD38-pretargeted radioimmunotherapy for plasma cell malignancies, Cancer Res, 74 (2014) 1179-1189.

[56] K. Subbiah, D.K. Hamlin, J.M. Pagel, D.S. Wilbur, D.L. Meyer, D.B. Axworthy, R.W. Mallett, L.J. Theodore, P.S. Stayton, O.W. Press, Comparison of immunoscintigraphy, efficacy, and toxicity of conventional and pretargeted radioimmunotherapy in CD20-expressing human lymphoma xenografts, J Nucl Med, 44 (2003) 437445.

[57] M. Zhang, Z. Zhang, K. Garmestani, J. Schultz, D.B. Axworthy, C.K. Goldman, M.W. Brechbiel, J.A. Carrasquillo, T.A. Waldmann, Pretarget radiotherapy with an anti-CD25 antibody-streptavidin fusion protein was effective in therapy of leukemia/lymphoma xenografts, Proc Natl Acad Sci U S A, 100 (2003) 1891-1895. 
[58] D.B. Axworthy, J.M. Reno, M.D. Hylarides, R.W. Mallett, L.J. Theodore, L.M. Gustavson, F. Su, L.J. Hobson, P.L. Beaumier, A.R. Fritzberg, Cure of human carcinoma xenografts by a single dose of pretargeted yttrium-90 with negligible toxicity, Proc Natl Acad Sci U S A, 97 (2000) 1802-1807.

[59] A. Forero, P.L. Weiden, J.M. Vose, S.J. Knox, A.F. LoBuglio, J. Hankins, M.L. Goris, V.J. Picozzi, D.B. Axworthy, H.B. Breitz, R.B. Sims, R.G. Ghalie, S. Shen, R.F. Meredith, Phase 1 trial of a novel anti-CD20 fusion protein in pretargeted radioimmunotherapy for B-cell non-Hodgkin lymphoma, Blood, 104 (2004) 227-236. [60] P.L. Weiden, H.B. Breitz, O. Press, J.W. Appelbaum, J.K. Bryan, S. Gaffigan, D. Stone, D. Axworthy, D. Fisher, J. Reno, Pretargeted radioimmunotherapy (PRIT) for treatment of non-Hodgkin's lymphoma (NHL): initial phase I/II study results, Cancer Biother Radiopharm, 15 (2000) 15-29.

[61] P.L. Weiden, H.B. Breitz, Pretargeted radioimmunotherapy (PRIT) for treatment of non-Hodgkin's lymphoma (NHL), Crit Rev Oncol Hematol, 40 (2001) 37-51.

[62] F. Kraeber-Bodere, S. Bardet, C.A. Hoefnagel, M.R. Vieira, J.P. Vuillez, A. Murat, T.C. Ferreira, M. Bardies, L. Ferrer, I. Resche, E. Gautherot, E. Rouvier, J. Barbet, J.F. Chatal, Radioimmunotherapy in medullary thyroid cancer using bispecific antibody and iodine 131-labeled bivalent hapten: preliminary results of a phase I/II clinical trial, Clin Cancer Res, 5 (1999) 3190s-3198s.

[63] F. Kraeber-Bodere, A. Faivre-Chauvet, C. Sai-Maurel, L. Campion, M. Fiche, E. Gautherot, J. Le Boterff, J. Barbet, J.F. Chatal, P. Thedrez, Toxicity and efficacy of radioimmunotherapy in carcinoembryonic antigenproducing medullary thyroid cancer xenograft: comparison of iodine 131-labeled $F\left(a^{\prime}\right) 2$ and pretargeted bivalent hapten and evaluation of repeated injections, Clin Cancer Res, 5 (1999) 3183s-3189s.

[64] F. Kraeber-Bodere, C. Rousseau, C. Bodet-Milin, L. Ferrer, A. Faivre-Chauvet, L. Campion, J.P. Vuillez, A. Devillers, C.H. Chang, D.M. Goldenberg, J.F. Chatal, J. Barbet, Targeting, toxicity, and efficacy of 2-step, pretargeted radioimmunotherapy using a chimeric bispecific antibody and 131I-labeled bivalent hapten in a phase I optimization clinical trial, J Nucl Med, 47 (2006) 247-255.

[65] A.C. Society, Cancer Facts \& Figures, in, American Cancer Society, Atlanta, 2015.

[66] S.M. Swain, S.B. Kim, J. Cortes, J. Ro, V. Semiglazov, M. Campone, E. Ciruelos, J.M. Ferrero, A.

Schneeweiss, A. Knott, E. Clark, G. Ross, M.C. Benyunes, J. Baselga, Pertuzumab, trastuzumab, and docetaxel for HER2-positive metastatic breast cancer (CLEOPATRA study): overall survival results from a randomised, doubleblind, placebo-controlled, phase 3 study, Lancet Oncol, 14 (2013) 461-471.

[67] Y. Koyama, T. Barrett, Y. Hama, G. Ravizzini, P.L. Choyke, H. Kobayashi, In vivo molecular imaging to diagnose and subtype tumors through receptor-targeted optically labeled monoclonal antibodies, Neoplasia, 9 (2007) 1021-1029.

[68] J.B. Haun, N.K. Devaraj, S.A. Hilderbrand, H. Lee, R. Weissleder, Bioorthogonal chemistry amplifies nanoparticle binding and enhances the sensitivity of cell detection, Nat Nanotechnol, 5 (2010) 660-665.

[69] M.R. Karver, R. Weissleder, S.A. Hilderbrand, Bioorthogonal reaction pairs enable simultaneous, selective, multi-target imaging, Angew Chem Int Ed Engl, 51 (2012) 920-922.

[70] B.A. Khaw, K.S. Gada, V. Patil, R. Panwar, S. Mandapati, A. Hatefi, S. Majewski, A. Weisenberger, Bispecific antibody complex pre-targeting and targeted delivery of polymer drug conjugates for imaging and therapy in dual human mammary cancer xenografts: targeted polymer drug conjugates for cancer diagnosis and therapy, Eur J Nucl Med Mol Imaging, 41 (2014) 1603-1616.

[71] J.J. Mulvey, C.H. Villa, M.R. McDevitt, F.E. Escorcia, E. Casey, D.A. Scheinberg, Self-assembly of carbon nanotubes and antibodies on tumours for targeted amplified delivery, Nat Nanotechnol, 8 (2013) 763-771.

[72] D.T. Reardan, C.F. Meares, D.A. Goodwin, M. McTigue, G.S. David, M.R. Stone, J.P. Leung, R.M.

Bartholomew, J.M. Frincke, Antibodies against metal chelates, Nature, 316 (1985) 265-268.

[73] D.A. Goodwin, C.F. Meares, M.J. McCall, M. McTigue, W. Chaovapong, Pre-targeted immunoscintigraphy of murine tumors with indium-111-labeled bifunctional haptens, J Nucl Med, 29 (1988) 226-234.

[74] H. Karacay, R.M. Sharkey, W.J. McBride, G.L. Griffiths, Z. Qu, K. Chang, H.J. Hansen, D.M. Goldenberg, Pretargeting for Cancer Radioimmunotherapy with Bispecific Antibodies: $\square$ Role of the Bispecific Antibody's Valency for the Tumor Target Antigen, Bioconjug Chem, 13 (2002) 1054-1070.

[75] F.G. van Schaijk, E. Oosterwijk, J.D. Molkenboer-Kuenen, A.C. Soede, B.J. McBride, D.M. Goldenberg, W.J.G. Oyen, F.H.M. Corstens, O.C. Boerman, Pretargeting with Bispecific Anti-Renal Cell Carcinoma x AntiDTPA(In) Antibody in 3 RCC Models, J Nucl Med, 46 (2005) 495-501.

[76] J.F. Gestin, A. Loussouarn, M. Bardies, E. Gautherot, A. Gruaz-Guyon, C. Sai-Maurel, J. Barbet, C. Curtet, J.F. Chatal, A. Faivre-Chauvet, Two-step targeting of xenografted colon carcinoma using a bispecific antibody and 188Re-labeled bivalent hapten: biodistribution and dosimetry studies, J Nucl Med, 42 (2001) 146-153. 
[77] M. Hillairet de Boisferon, O. Raguin, M. Dussaillant, W. Rostène, J. Barbet, A. Gruaz-Guyon, Enhanced Targeting Specificity to Tumor Cells by Simultaneous Recognition of Two Antigens, Bioconjug Chem, 11 (2000) 452-460.

[78] W.J. McBride, P. Zanzonico, R.M. Sharkey, C. Noren, H. Karacay, E.A. Rossi, M.J. Losman, P.Y. Brard, C.H. Chang, S.M. Larson, D.M. Goldenberg, Bispecific antibody pretargeting PET (immunoPET) with an 124I-labeled hapten-peptide, J Nucl Med, 47 (2006) 1678-1688.

[79] M.V. Pimm, R.A. Robins, M.J. Embleton, E. Jacobs, A.J. Markham, A. Charleston, R.W. Baldwin, A

bispecific monoclonal antibody against methotrexate and a human tumour associated antigen augments cytotoxicity of methotrexate-carrier conjugate, Br J Cancer, 61 (1990) 508-513.

[80] S. Yoon, Y.H. Kim, S.H. Kang, S.K. Kim, H.K. Lee, H. Kim, J. Chung, I.H. Kim, Bispecific Her2 x cotinine antibody in combination with cotinine-(histidine)2-iodine for the pre-targeting of Her2-positive breast cancer xenografts, J Cancer Res Clin Oncol, 140 (2014) 227-233.

[81] K.S. Gada, V. Patil, R. Panwar, A. Hatefi, B.A. Khaw, Bispecific antibody complex pre-targeted delivery of polymer-drug conjugates for cancer therapy, Drug Deliv Transl Res, 2 (2012) 65-76.

[82] J.M. Le Doussal, M. Martin, E. Gautherot, M. Delaage, J. Barbet, In vitro and in vivo targeting of radiolabeled monovalent and divalent haptens with dual specificity monoclonal antibody conjugates: enhanced divalent hapten affinity for cell-bound antibody conjugate, J Nucl Med, 30 (1989) 1358-1366.

[83] E. Janevik-Ivanovska, E. Gautherot, M. Hillairet de Boisferon, M. Cohen, G. Milhaud, A. Tartar, W. Rostene, J. Barbet, A. Gruaz-Guyon, Bivalent hapten-bearing peptides designed for iodine-131 pretargeted radioimmunotherapy, Bioconjug Chem, 8 (1997) 526-533.

[84] O.C. Boerman, M.H. Kranenborg, E. Oosterwijk, G.L. Griffiths, W.J. McBride, W.J. Oyen, M. de Weijert, J. Oosterwijk-Wakka, H.J. Hansen, F.H. Corstens, Pretargeting of renal cell carcinoma: improved tumor targeting with a bivalent chelate, Cancer Res, 59 (1999) 4400-4405.

[85] H.P. Lesch, M.U. Kaikkonen, J.T. Pikkarainen, S. Yla-Herttuala, Avidin-biotin technology in targeted therapy, Expert Opin Drug Deliv, 7 (2010) 551-564.

[86] M.V. Pimm, H.F. Fells, A.C. Perkins, R.W. Baldwin, Iodine-131 and indium-111 labelled avidin and streptavidin for pre-targetted immunoscintigraphy with biotinylated anti-tumour monoclonal antibody, Nucl Med Commun, 9 (1988) 931-941.

[87] J.R. Newton-Northup, S.D. Figueroa, T.P. Quinn, S.L. Deutscher, Bifunctional phage-based pretargeted imaging of human prostate carcinoma, Nucl Med Biol, 36 (2009) 789-800.

[88] S.J. Knox, M.L. Goris, M. Tempero, P.L. Weiden, L. Gentner, H. Breitz, G.P. Adams, D. Axworthy, S. Gaffigan, K. Bryan, D.R. Fisher, D. Colcher, I.D. Horak, L.M. Weiner, Phase II trial of yttrium-90-DOTA-biotin pretargeted by NR-LU-10 antibody/streptavidin in patients with metastatic colon cancer, Clin Cancer Res, 6 (2000) 406-414.

[89] Y. Lin, J.M. Pagel, D. Axworthy, A. Pantelias, N. Hedin, O.W. Press, A genetically engineered anti-CD45 single-chain antibody-streptavidin fusion protein for pretargeted radioimmunotherapy of hematologic malignancies, Cancer Res, 66 (2006) 3884-3892.

[90] N.K. Cheung, S. Modak, Y. Lin, H. Guo, P. Zanzonico, J. Chung, Y. Zuo, J. Sanderson, S. Wilbert, L.J. Theodore, D.B. Axworthy, S.M. Larson, Single-chain Fv-streptavidin substantially improved therapeutic index in multistep targeting directed at disialoganglioside GD2, J Nucl Med, 45 (2004) 867-877.

[91] J.M. Pagel, Y. Lin, N. Hedin, A. Pantelias, D. Axworthy, D. Stone, D.K. Hamlin, D.S. Wilbur, O.W. Press, Comparison of a tetravalent single-chain antibody-streptavidin fusion protein and an antibody-streptavidin chemical conjugate for pretargeted anti-CD20 radioimmunotherapy of B-cell lymphomas, Blood, 108 (2006) 328-336. [92] M.D. Stachler, I. Chen, A.Y. Ting, J.S. Bartlett, Site-specific modification of AAV vector particles with biophysical probes and targeting ligands using biotin ligase, Mol Ther, 16 (2008) 1467-1473.

[93] H.P. Kalofonos, M. Rusckowski, D.A. Siebecker, G.B. Sivolapenko, D. Snook, J.P. Lavender, A.A. Epenetos, D.J. Hnatowich, Imaging of tumor in patients with indium-111-labeled biotin and streptavidin-conjugated antibodies: preliminary communication, J Nucl Med, 31 (1990) 1791-1796.

[94] K. Yumura, M. Ui, H. Doi, T. Hamakubo, T. Kodama, K. Tsumoto, A. Sugiyama, Mutations for decreasing the immunogenicity and maintaining the function of core streptavidin, Protein Sci, 22 (2013) 213-221.

[95] D.L. Meyer, J. Schultz, Y. Lin, A. Henry, J. Sanderson, J.M. Jackson, S. Goshorn, A.R. Rees, S.S. Graves, Reduced antibody response to streptavidin through site-directed mutagenesis, Protein Sci, 10 (2001) 491-503. [96] M. Rusckowski, M. Fogarasi, B. Fritz, D.J. Hnatowich, Effect of endogenous biotin on the applications of streptavidin and biotin in mice, Nucl Med Biol, 24 (1997) 263-268. 
[97] K.J. Hamblett, O.W. Press, D.L. Meyer, D.K. Hamlin, D. Axworthy, D.S. Wilbur, P.S. Stayton, Role of biotinbinding affinity in streptavidin-based pretargeted radioimmunotherapy of lymphoma, Bioconjug Chem, 16 (2005) 131-138.

[98] D.S. Wilbur, S.I. Park, M.K. Chyan, F. Wan, D.K. Hamlin, J. Shenoi, Y. Lin, S.M. Wilbur, F. Buchegger, A. Pantelias, J.M. Pagel, O.W. Press, Design and synthesis of bis-biotin-containing reagents for applications utilizing monoclonal antibody-based pretargeting systems with streptavidin mutants, Bioconjug Chem, 21 (2010) 1225-1238. [99] M. Steiner, K. Gutbrodt, N. Krall, D. Neri, Tumor-targeting antibody-anticalin fusion proteins for in vivo pretargeting applications, Bioconjug Chem, 24 (2013) 234-241.

[100] J.C. Knight, M. Mosley, M.R. Stratford, H.T. Uyeda, H.A. Benink, M. Cong, F. Fan, S. Faulkner, B. Cornelissen, Development of an enzymatic pretargeting strategy for dual-modality imaging, Chem Commun (Camb), 51 (2015) 4055-4058.

[101] G. Liu, S. Dou, Y. Liu, Y. Wang, M. Rusckowski, D.J. Hnatowich, 90Y labeled phosphorodiamidate morpholino oligomer for pretargeting radiotherapy, Bioconjug Chem, 22 (2011) 2539-2545.

[102] G. Liu, S. Dou, P.H. Pretorius, X. Liu, L. Chen, M. Rusckowski, D.J. Hnatowich, Tumor pretargeting in mice using MORF conjugated CC49 antibody and radiolabeled complimentary cMORF effector, Q J Nucl Med Mol Imaging, 54 (2010) 333-340.

[103] G. Liu, C. Liu, S. Zhang, J. He, N. Liu, S. Gupta, M. Rusckowski, D.J. Hnatowich, Investigations of 99mTc morpholino pretargeting in mice, Nucl Med Commun, 24 (2003) 697-705.

[104] G. Liu, J. He, S. Dou, S. Gupta, M. Rusckowski, D.J. Hnatowich, Further investigations of morpholino pretargeting in mice--establishing quantitative relations in tumor, Eur J Nucl Med Mol Imaging, 32 (2005) 11151123.

[105] J. He, G. Liu, S. Gupta, Y. Zhang, M. Rusckowski, D.J. Hnatowich, Amplification Targeting: A Modified Pretargeting Approach with Potential for Signal Amplification-Proof of a Concept, J Nucl Med, 45 (2004) 10871095.

[106] R.M. Sharkey, D.M. Goldenberg, Cancer radioimmunotherapy, Immunotherapy, 3 (2011) 349-370.

[107] J. He, Y. Wang, S. Dou, X. Liu, S. Zhang, G. Liu, D. Hnatowich, Affinity enhancement pretargeting: synthesis and testing of a 99mTc-labeled bivalent MORF, Mol Pharm, 7 (2010) 1118-1124.

[108] L. Carroll, H.L. Evans, E.O. Aboagye, A.C. Spivey, Bioorthogonal chemistry for pre-targeted molecular imaging--progress and prospects, Org Biomol Chem, 11 (2013) 5772-5781.

[109] J.C. Knight, B. Cornelissen, Bioorthogonal chemistry: implications for pretargeted nuclear (PET/SPECT) imaging and therapy, American Journal of Nuclear Medicine and Molecular Imaging, 4 (2014) 96-113.

[110] R. Rossin, P. Renart Verkerk, S.M. van den Bosch, R.C.M. Vulders, I. Verel, J. Lub, M.S. Robillard, In Vivo Chemistry for Pretargeted Tumor Imaging in Live Mice, Angewandte Chemie International Edition, 49 (2010) 3375-3378.

[111] R. Rossin, S.M. van Duijnhoven, T. Lappchen, S.M. van den Bosch, M.S. Robillard, Trans-cyclooctene tag with improved properties for tumor pretargeting with the diels-alder reaction, Mol Pharm, 11 (2014) 3090-3096. [112] B.M. Zeglis, K.K. Sevak, T. Reiner, P. Mohindra, S.D. Carlin, P. Zanzonico, R. Weissleder, J.S. Lewis, A pretargeted PET imaging strategy based on bioorthogonal Diels-Alder click chemistry, J Nucl Med, 54 (2013) 1389-1396.

[113] S.B. Lee, H.L. Kim, H.-J. Jeong, S.T. Lim, M.-H. Sohn, D.W. Kim, Mesoporous Silica Nanoparticle Pretargeting for PET Imaging Based on a Rapid Bioorthogonal Reaction in a Living Body, Angewandte Chemie International Edition, 52 (2013) 10549-10552.

[114] J. Gunn, S.I. Park, O. Veiseh, O.W. Press, M. Zhang, A pretargeted nanoparticle system for tumor cell labeling, Mol Biosyst, 7 (2011) 742-748.

[115] T.W. Chu, J. Yang, R. Zhang, M. Sima, J. Kopecek, Cell surface self-assembly of hybrid nanoconjugates via oligonucleotide hybridization induces apoptosis, ACS Nano, 8 (2014) 719-730.

[116] L. Nobs, F. Buchegger, R. Gurny, E. Allemann, Biodegradable nanoparticles for direct or two-step tumor immunotargeting, Bioconjug Chem, 17 (2006) 139-145.

[117] J. Bushman, A. Vaughan, L. Sheihet, Z. Zhang, M. Costache, J. Kohn, Functionalized nanospheres for targeted delivery of paclitaxel, J Control Release, 171 (2013) 315-321.

[118] M. Pulkkinen, J. Pikkarainen, T. Wirth, T. Tarvainen, V. Haapa-aho, H. Korhonen, J. Seppälä, K. Järvinen, Three-step tumor targeting of paclitaxel using biotinylated PLA-PEG nanoparticles and avidin-biotin technology: Formulation development and in vitro anticancer activity, European Journal of Pharmaceutics and Biopharmaceutics, 70 (2008) 66-74. 
[119] S.A. Longman, P.R. Cullis, L. Choi, G. de Jong, M.B. Bally, A two-step targeting approach for delivery of doxorubicin-loaded liposomes to tumour cells in vivo, Cancer Chemother Pharmacol, 36 (1995) 91-101.

[120] S.H. Frost, H. Jensen, S. Lindegren, In vitro evaluation of avidin antibody pretargeting using 211At-labeled and biotinylated poly-L-lysine as effector molecule, Cancer, 116 (2010) 1101-1110.

[121] Y. Cao, M.R. Suresh, Bispecific MAb aided liposomal drug delivery, J Drug Target, 8 (2000) 257-266.

[122] M.K. Rahim, R. Kota, J.B. Haun, Enhancing reactivity for bioorthogonal pretargeting by unmasking antibody-conjugated trans-cyclooctenes, Bioconjug Chem, 26 (2015) 352-360.

[123] I. Navarro-Teulon, C. Lozza, A. Pelegrin, E. Vives, J.P. Pouget, General overview of radioimmunotherapy of solid tumors, Immunotherapy, 5 (2013) 467-487.

[124] N. Sato, R. Hassan, D.B. Axworthy, K.J. Wong, S. Yu, L.J. Theodore, Y. Lin, L. Park, M.W. Brechbiel, I. Pastan, C.H. Paik, J.A. Carrasquillo, Pretargeted radioimmunotherapy of mesothelin-expressing cancer using a tetravalent single-chain Fv-streptavidin fusion protein, J Nucl Med, 46 (2005) 1201-1209.

[125] D.M. Goldenberg, E.A. Rossi, R.M. Sharkey, W.J. McBride, C.H. Chang, Multifunctional antibodies by the

Dock-and-Lock method for improved cancer imaging and therapy by pretargeting, J Nucl Med, 49 (2008) 158-163.

[126] R.M. Sharkey, H. Karacay, S. Litwin, E.A. Rossi, W.J. McBride, C.H. Chang, D.M. Goldenberg, Improved

therapeutic results by pretargeted radioimmunotherapy of non-Hodgkin's lymphoma with a new recombinant, trivalent, anti-CD20, bispecific antibody, Cancer Res, 68 (2008) 5282-5290.

[127] R.M. Sharkey, F.J. Primus, D.M. Goldenberg, Second antibody clearance of radiolabeled antibody in cancer radioimmunodetection, Proc Natl Acad Sci U S A, 81 (1984) 2843-2846.

[128] E. Mirallié, C. Saï-Maurel, A. Faivre-Chauvet, N. Regenet, C.-H. Chang, D. Goldenberg, J.-F. Chatal, J. Barbet, P. Thedrez, Improved pretargeted delivery of radiolabelled hapten to human tumour xenograft in mice by avidin chase of circulating bispecific antibody, Eur J Nucl Med Mol Imaging, 32 (2005) 901-909.

[129] H.B. Breitz, P.L. Weiden, P.L. Beaumier, D.B. Axworthy, C. Seiler, F.M. Su, S. Graves, K. Bryan, J.M. Reno, Clinical optimization of pretargeted radioimmunotherapy with antibody-streptavidin conjugate and 90Y-DOTAbiotin, J Nucl Med, 41 (2000) 131-140.

[130] G. Liu, S. Dou, X. Chen, L. Chen, X. Liu, M. Rusckowski, D.J. Hnatowich, Adding a clearing agent to pretargeting does not lower the tumor accumulation of the effector as predicted, Cancer Biother Radiopharm, 25 (2010) 757-762.

[131] G. Paganelli, C. Grana, M. Chinol, M. Cremonesi, C. De Cicco, F. De Braud, C. Robertson, S. Zurrida, C. Casadio, S. Zoboli, A.G. Siccardi, U. Veronesi, Antibody-guided three-step therapy for high grade glioma with yttrium-90 biotin, Eur J Nucl Med, 26 (1999) 348-357.

[132] C. Grana, M. Chinol, C. Robertson, C. Mazzetta, M. Bartolomei, C. De Cicco, M. Fiorenza, M. Gatti, P. Caliceti, G. Paganelli, Pretargeted adjuvant radioimmunotherapy with yttrium-90-biotin in malignant glioma patients: a pilot study, Br J Cancer, 86 (2002) 207-212.

[133] D.M. Goldenberg, J.F. Chatal, J. Barbet, O. Boerman, R.M. Sharkey, Cancer Imaging and Therapy with Bispecific Antibody Pretargeting, Update Cancer Ther, 2 (2007) 19-31.

[134] R. Sabatier, A. Goncalves, F. Bertucci, Personalized medicine: present and future of breast cancer management, Crit Rev Oncol Hematol, 91 (2014) 223-233.

[135] A. Pantelias, J.M. Pagel, N. Hedin, L. Saganic, S. Wilbur, D.K. Hamlin, D.S. Wilbur, Y. Lin, D. Stone, D. Axworthy, A.K. Gopal, O.W. Press, Comparative biodistributions of pretargeted radioimmunoconjugates targeting CD20, CD22, and DR molecules on human B-cell lymphomas, Blood, 109 (2007) 4980-4987.

[136] T.E. Witzig, L.I. Gordon, F. Cabanillas, M.S. Czuczman, C. Emmanouilides, R. Joyce, B.L. Pohlman, N.L. Bartlett, G.A. Wiseman, N. Padre, A.J. Grillo-Lopez, P. Multani, C.A. White, Randomized controlled trial of yttrium-90-labeled ibritumomab tiuxetan radioimmunotherapy versus rituximab immunotherapy for patients with relapsed or refractory low-grade, follicular, or transformed B-cell non-Hodgkin's lymphoma, J Clin Oncol, 20 (2002) 2453-2463.

[137] M. Jain, N. Kamal, S.K. Batra, Engineering antibodies for clinical applications, Trends Biotechnol, 25 (2007) 307-316. 


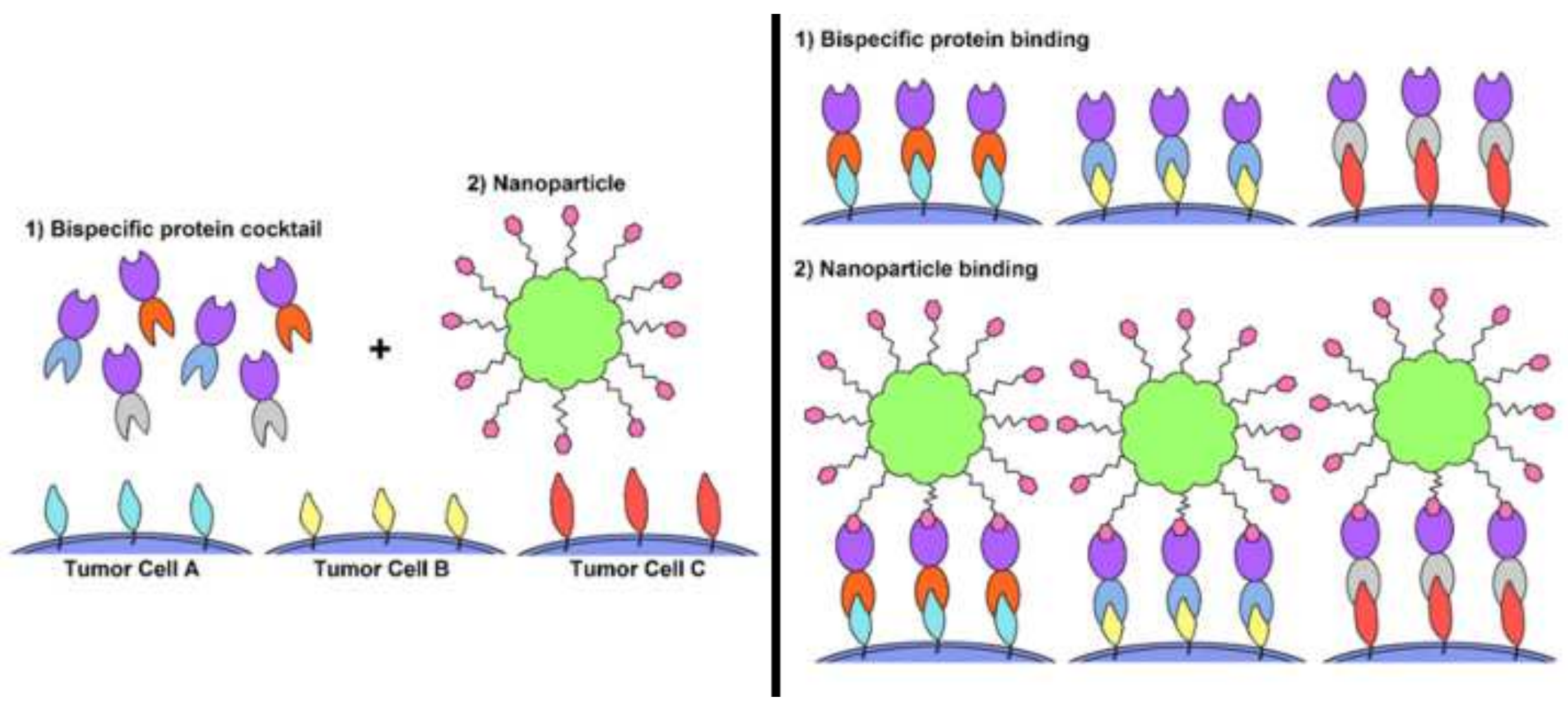

